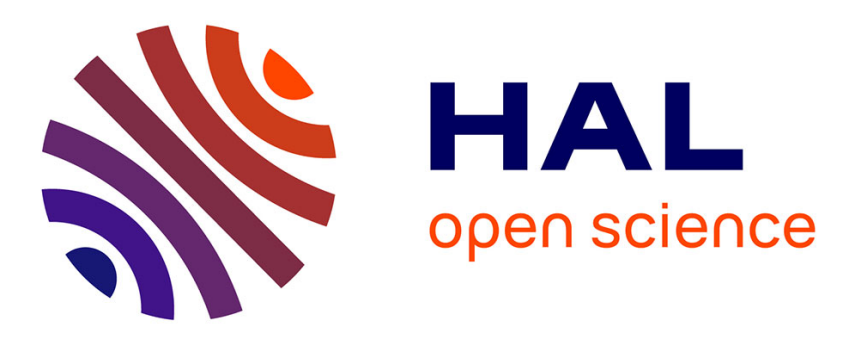

\title{
Valence-bond crystal and lattice distortions in a pyrochlore antiferromagnet with orbital degeneracy
}

Sergio Di Matteo, G Jackeli, N B Perkins

\section{To cite this version:}

Sergio Di Matteo, G Jackeli, N B Perkins. Valence-bond crystal and lattice distortions in a pyrochlore antiferromagnet with orbital degeneracy. Physical Review B: Condensed Matter and Materials Physics (1998-2015), 2005, 72, 10.1103/physrevb.72.024431 . hal-03110676

\section{HAL Id: hal-03110676 \\ https://hal.science/hal-03110676}

Submitted on 14 Jan 2021

HAL is a multi-disciplinary open access archive for the deposit and dissemination of scientific research documents, whether they are published or not. The documents may come from teaching and research institutions in France or abroad, or from public or private research centers.
L'archive ouverte pluridisciplinaire HAL, est destinée au dépôt et à la diffusion de documents scientifiques de niveau recherche, publiés ou non, émanant des établissements d'enseignement et de recherche français ou étrangers, des laboratoires publics ou privés. 


\title{
Valence-bond crystal and lattice distortions in a pyrochlore antiferromagnet with orbital degeneracy
}

\author{
S. Di Matteo, ${ }^{1,2}$ G. Jackeli, $3,4, *$ and N. B. Perkins ${ }^{1,5,6}$ \\ ${ }^{1}$ Laboratori Nazionali di Frascati INFN, via E. Fermi 40, C.P. 13, I-00044 Frascati (Roma) Italy \\ ${ }^{2}$ Dipartimento di Fisica, Università di Roma III, via della Vasca Navale 84, I-00146 Roma, Italy \\ ${ }^{3}$ Ecole Polytechnique Fédérale de Lausanne, Institute for Theoretical Physics, CH-1025, Lausanne, Switzerland \\ ${ }^{4}$ Institut Laue Langevin, B. P. 156, F-38042, Grenoble, France \\ ${ }^{5}$ Bogoliubov Laboratory of Theoretical Physics, JINR, 141980, Dubna, Russia \\ ${ }^{6}$ Max Planck Institute for the Physics of Complex Systems, Noethnitzer Strasse 38, D-01187 Dresden, Germany
}

(Received 23 December 2004; revised manuscript received 4 April 2005; published 14 July 2005)

\begin{abstract}
We discuss the ground state properties of a spin-1/2 magnetic ion with threefold $t_{2 g}$ orbital degeneracy on a highly frustrated pyrochlore lattice, like $\mathrm{Ti}^{3+}$ ion in $\mathrm{B}$-spinel $\mathrm{MgTi}_{2} \mathrm{O}_{4}$. We formulate an effective spin-orbital Hamiltonian and study its low energy sector by constructing several exact eigenstates in the limit of vanishing Hund's coupling. We find that orbital degrees of freedom modulate the spin-exchange energies, release the infinite spin degeneracy of pyrochlore structure, and drive the system to a nonmagnetic spin-singlet manifold. The latter is a collection of spin-singlet dimers and is, however, highly degenerate with respect to dimer orientations. This "orientational" degeneracy is then lifted by a magneto-elastic interaction that optimizes the previous energy gain by distorting the bonds in suitable directions and leading to a tetragonal phase. In this way a valence bond crystal state is formed, through the condensation of dimers along helical chains running around the tetragonal $c$ axis, as actually observed in $\mathrm{MgTi}_{2} \mathrm{O}_{4}$. The orbitally ordered pattern in the dimerized phase is predicted to be of ferro type along the helices and of antiferro type between them. Finally, through analytical considerations as well as numerical ab initio simulations, we predict a possible experimental tool for the observation of such an orbital ordering, through resonant x-ray scattering.
\end{abstract}

DOI: $10.1103 /$ PhysRevB.72.024431

PACS number(s): 75.10.Jm, 75.30.Et

\section{INTRODUCTION}

A frustrated antiferromagnet is characterized by the topology of the underlying lattice and/or by the presence of competing interactions that preclude every pairwise magnetic interaction to be satisfied at the same time. Such physical systems have recently attracted a wide interest, ${ }^{1}$ due to the concept of "macroscopic" degeneracy, namely the existence of a huge number of states with the same energy. This degeneracy in the ground state manifold can be usually removed through a large variety of effects, like order-out-ofdisorder mechanisms ${ }^{2}$ by thermal or quantum fluctuations, or spin-Peierls like symmetry-lowering transitions. ${ }^{3,4}$ In some highly frustrated models, like the nearest-neighbor Heisenberg antiferromagnet on pyrochlore lattice, the former mechanisms are inactive ${ }^{5}$ and such a spin system would remain liquid down to the lowest temperatures, ${ }^{6}$ unless magnetoelastic couplings induce a symmetry-breaking transition. Yet, in real compounds geometrical frustration can also be partially or fully released when magnetic ions forming a frustrated lattice possess an orbital degeneracy. Typically this happens in transition metal ions with orbitally degenerate partly filled $d$ levels. The physical behavior of such systems is expected to be drastically different from that of pure spin models, as the occurrence of an orbital ordering (OO) can modulate the spin exchange and lift the geometrical degeneracy of the underlying lattice. Indeed, a variety of novel phases driven by orbital degrees of freedom can be stabilized in this way. Among known examples are vanadium $d^{2}$ compounds with frustrated lattices, where the orbital order is shown to induce a spin-singlet ground state without any long-range magnetic order for triangular lattice, ${ }^{7}$ or a spin ordered one, for pyrochlore lattice. ${ }^{8}$

In this paper we study a system with threefold orbitally degenerate $S=1 / 2$ magnetic ions in a corner-sharing tetrahedral (pyrochlore) lattice. Our work is motivated by the very recent synthesis ${ }^{9}$ and interesting experimental data on $B$-spinel $\mathrm{MgTi}_{2} \mathrm{O}_{4},{ }^{9,10}$ a $d^{1}$-type transition metal oxide. Here magnetically active $\mathrm{Ti}^{3+}$ ions form a pyrochlore lattice and are characterized by a single electron in a $d$ shell. The crystal field of the oxygen octahedron surrounding each $\mathrm{Ti}$ ion splits this $d$-level into a high energy doublet $e_{g}$ and a low energy triplet $t_{2 g}$ in which the $d$ electron resides. ${ }^{11}$ The ground state of a spin $1 / 2 \mathrm{Ti}^{3+}$ ion is thus threefold orbitally degenerate. The recent experiments on $\mathrm{MgTi}_{2} \mathrm{O}_{4}$ have shown that this compound undergoes a metal-to-insulator transition on cooling below $260 \mathrm{~K}$, with an associated cubic-to-tetragonal lowering of the symmetry. ${ }^{9}$ At the transition the magnetic susceptibility continuously decreases and saturates, in the insulating phase, to a value which is anomalously small for spin 1/2 local moments: for this reason the insulating phase has been interpreted as a spin-singlet phase. Subsequent synchrotron and neutron powder diffraction experiments have revealed that the low-temperature crystal structure is made of alternating short and long $\mathrm{Ti}-\mathrm{Ti}$ bonds forming a helix about the tetragonal $c$ axis. ${ }^{10}$ These findings have suggested a removal of the pyrochlore degeneracy by a one-dimensional (1D) helical dimerization of the spin pattern, with spinsinglets (dimers) located at short bonds. This phase can be regarded as a valence bond crystal (VBC) since the long- 
range order of spin-singlets extends throughout the whole pyrochlore lattice. Indeed, due to the orbital degeneracy, various types of spin-singlet phases, such as resonating valence bond (RVB) and VBC states, can be formed even for unfrustrated cubic lattice. ${ }^{12}$

The aim of the present work is to discuss the microscopic mechanism behind the realization of this unusual and intriguing VBC structure on the pyrochlore lattice. We argue that the key role in this mechanism is played by orbital degeneracy: The orbital degree of freedom does modulate spin exchange energies, thus removing the infinite spin degeneracy, characteristic of pyrochlore structures, and drives the system to a nonmagnetic spin-singlet state. This latter is a collection of spin-singlet dimers with a residual macroscopic degeneracy of orientational character. The residual degeneracy is then lifted by a magnetoelastic interaction, which optimizes superexchange energy gain by distorting each tetrahedron in such a way as to lead to the experimentally observed helical pattern in $\mathrm{MgTi}_{2} \mathrm{O}_{4}$. We also find that the helical dimerized state is accompanied by a peculiar orbital pattern in which orbital order is of ferrotype along the helices and of antiferrotype between them.

Moreover, we can show how to identify such an orbital ordering experimentally, by means of resonant x-ray scattering (RXS). In fact, differently from what happens in the case of manganites, ${ }^{13-16}$ where the ratio between the OO-induced and Jahn-Teller (JT)-induced effects is about 1/10 in amplitude, for $\mathrm{MgTi}_{2} \mathrm{O}_{4}$ this ratio is about $1 / 3$, due to the less distorted oxygen octahedra, as well as to the presence of $t_{2 g}$ electrons instead of $e_{g}$, which couple less to the oxygen environment. Such a reduced ratio allows a subtle interference effect between $\mathrm{OO}$ and JT terms, which gives rise to an increase of the signal by a factor of about 1.7. We have performed a detailed analytical analysis as well as an $a b$ initio numerical simulation to suggest some experiments in this direction.

In more detail, the paper is organized as follows: in Sec. II we derive an effective Kugel-Khomskii ${ }^{17}$ model Hamiltonian, and deduce its possible low-energy states on the pyrochlore lattice. We then introduce the spin-singlet ground state manifold and discuss its degeneracy due to the dimer coverings of the pyrochlore lattice. In Sec. III we consider the magnetoelastic interactions of the pyrochlore lattice and analyze the effects of the coupling of bond distortions with the orbitally driven exchange modulation, first qualitatively, and then quantitatively, underlining the influence of the spinsinglet phase on the strength of such a distortion. In Sec. IV the effect of an applied magnetic field is briefly discussed, and, finally, in Sec. V we analyze the crystal structure and the orbital symmetry of the tetragonal phase, deriving the analytic expression for the measurable quantities in a RXS experiment. We also perform a series of numerical ab initio simulations by means of the finite difference method, implemented in the FDMNES package, ${ }^{18}$ in order to propose a possible experiment to detect the orbital pattern. To faciliate the reader, two Appendices, A and B, are given for technical details.

Part of the results presented here were already announced in a previous short communication. ${ }^{19}$

\section{MODEL AND FORMALISM}

\section{A. Effective spin-orbital Hamiltonian}

Here we discuss the superexchange spin-orbital Hamiltonian for threefold orbitally degenerate $d^{1}$ ions on a pyrochlore lattice. We assume that the insulating phase of $\mathrm{MgTi}_{2} \mathrm{O}_{4}$ is of Mott-Hubbard type and can thus be described by the Kugel-Khomskii model. ${ }^{17}$ We consider the system in its cubic structure and look for possible instabilities towards symmetry reductions.

The relevant electronic degrees of freedom are described by spin $S=1 / 2$ and pseudospin $\tau=1$ operators. This latter labels the orbital occupancies of $t_{2 g}$ orbitals, $(|\alpha \beta\rangle$ $=|x y\rangle,|x z\rangle,|y z\rangle)$, with the correspondence: $\tau^{z}=-1 \rightarrow|y z\rangle, \tau^{z}$ $=0 \rightarrow|x y\rangle$, and $\tau^{z}=1 \rightarrow|x z\rangle$. Our parameters are the nearestneighbor (NN) electron-hopping matrix $\hat{t}$, defined in Appendix A [Eq. (A1)], the Coulomb on-site repulsions $U_{1}$ (within the same orbital) and $U_{2}$ (among different orbitals), and the Hund's exchange $J_{H}$. For $t_{2 g}$ wave functions the relation $U_{1}=U_{2}+2 J_{H}$ holds due to cubic symmetry in real space.

Considering the pyrochlore structure the effective Hamiltonian can be simplified by retaining only the leading hopping parameter, $t$, due to the $\mathrm{NN} d d \sigma$ overlap and neglecting the smaller $d d \pi$ and $d d \delta$ contributions. This is justified by the fact that the transfer integrals due to the $\pi$ and $\delta$ bonding are, respectively, around $1 / 10$ and $1 / 3$ of that of $\sigma$ bonding. ${ }^{20}$ The major advantage of this simplification is that $d d \sigma$ overlap in the $\alpha \beta$ plane connects only the corresponding orbitals of the same $\alpha \beta$ type. Thus, the total number of electrons in each orbital state is a conserved quantity and, therefore, the orbital part of the effective Hamiltonian (A2) becomes Ising-like (or better, a Potts $Z_{3}$-like), making it possible to get analytical results about the ground state. The spin-orbital Hamiltonian is presented in Appendix A in the most general form [see Eq. (A2)], when also $d d \pi$ and $d d \delta$ hopping elements are considered. Here we introduce the simplified version with only $d d \sigma$ hopping terms:

$$
\begin{aligned}
H_{\mathrm{eff}}= & -J_{1} \sum_{\langle i j\rangle}\left[\vec{S}_{i} \cdot \vec{S}_{j}+3 / 4\right] O_{i j}+J_{2} \sum_{\langle i j\rangle}\left[\vec{S}_{i} \cdot \vec{S}_{j}-1 / 4\right] O_{i j} \\
& +J_{3} \sum_{\langle i j\rangle}\left[\vec{S}_{i} \cdot \vec{S}_{j}-1 / 4\right] \widetilde{O}_{i j},
\end{aligned}
$$

where the sum is restricted to the NN sites on the pyrochlore lattice. The orbital contributions along the bond $i j$ in $\alpha \beta$-plane is given by

$$
\begin{gathered}
O_{i j}=P_{i, \alpha \beta}\left(1-P_{j, \alpha \beta}\right)+P_{j, \alpha \beta}\left(1-P_{i, \alpha \beta}\right), \\
\widetilde{O}_{i j}=P_{i, \alpha \beta} P_{j, \alpha \beta},
\end{gathered}
$$

where $P_{i, \alpha \beta}=T_{i}^{a a}$ stands for the projector on orbital state $|a\rangle$ $=|\alpha \beta\rangle$ and is defined in Appendix A.

The first and second terms in $H_{\text {eff }}$ (1) describe the ferromagnetic (FM) $J_{1}=t^{2} /\left(U_{2}-J_{H}\right)$ and the antiferromagnetic (AFM) $J_{2}=t^{2} /\left(U_{2}+J_{H}\right)$ interactions, respectively, and are active only when the two sites involved are occupied by different orbitals. The last term is AFM, with $J_{3}=\frac{4}{3} t^{2}\left[2 /\left(U_{2}+J_{H}\right)\right.$ $\left.+1 /\left(U_{2}+4 J_{H}\right)\right]$ and is nonzero only when the two sites have the same orbital occupancy. Parameters that play a role in the 


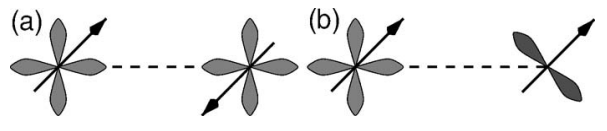

FIG. 1. The orbital arrangements on a bond: (a) bond $b_{0}$ with strong AFM coupling $\sim J$ and (b) bond $b_{1}$ with weak FM coupling $\sim \eta J$.

Hamiltonian (1) are $t \equiv t_{\sigma} \simeq 0.32 \mathrm{eV}, J_{H} \simeq 0.64 \mathrm{eV}$, and $U_{2}$ $\simeq 4.1 \mathrm{eV} .^{20,21}$ Thus $\eta=J_{H} / U_{2} \simeq 0.15 \ll 1$ and, just in order to present the results in a more transparent form, we expand the exchange energies around $\eta=0$. We get $J_{1} \simeq J(1+\eta), J_{2}$ $\simeq J(1-\eta)$, and $J_{3} \simeq 4 J(1-2 \eta)$, where $J=t^{2} / U_{2} \simeq 25 \mathrm{meV}$ represents the overall energy scale.

The realization of the spin-orbital model (1) on the pyrochlore lattice has an immediate and important consequence, namely, that only some bonds can contribute to the energy, depending on their orbital configuration. Indeed, every bond $i j$ in the $\alpha \beta$ plane has zero energy gain unless at least one of the two sites $i$ and $j$ has an occupied orbital of $\alpha \beta$ kind. The strength, as well as the sign, of spin-exchange energy associated with two NN sites $i$ and $j$ depends only on their orbital occupations and the direction of the $i j$ bond. We can thus classify tetrahedral bonds in four types:

(i) $b_{0}$, when both ions at sites $i$ and $j$ of the generic $\alpha \beta$ plane have $\alpha \beta$ orbital occupancy [Fig. 1(a)]. It is characterized by a Hamiltonian with strong AFM exchange $\sim J$ :

$$
H_{b_{0}}=-J(1-2 \eta)\left(1-4 \vec{S}_{i} \cdot \vec{S}_{j}\right) \text {. }
$$

(ii) $b_{1}$, if the two sites of bond $i j$ in the $\alpha \beta$ plane are occupied by one $\alpha \gamma$ and one $\alpha \beta$ orbital, $\gamma \neq \beta$ [Fig. 1(b)]. This bond has a weak FM exchange $\sim \eta J$ and the corresponding Hamiltonian is

$$
H_{b_{1}}=-J\left(1+\eta / 2+2 \eta \vec{S}_{i} \cdot \vec{S}_{j}\right) .
$$

(iii) $b_{2}$, with the two sites of bond $i j$ in the $\alpha \beta$ plane occupied by one $\alpha \gamma$ and one $\beta \gamma$ orbital. In this case there is no energy contribution: $H_{b_{2}}=0$.

(iv) $b_{3}$, if both sites of bond $i j$ in the $\alpha \beta$ plane are occupied by two $\alpha \gamma(\beta \gamma)$ orbitals. Also this bond does not contribute to the energy: $H_{b_{3}}=0$.

Thus, only $b_{0}$ and $b_{1}$ bonds have a binding energy and for any given orbital configuration on the pyrochlore lattice the Hamiltonian (1) is just a linear combination of $H_{b_{0}}$ and $H_{b_{1}}$. Moreover, the explicit form of Eqs. (3) and (4) implies that, in the limit $\eta \rightarrow 0$, a set of exact eigenstates of (1) can be constructed: in fact, orbital interactions are already diagonal, and, in this limit, the only Heisenberg term that survives is that of $H_{b_{0}}$. The latter, by definition, is active only along the direction connecting the two sites with equally filled $\alpha \beta$ orbitals. This implies that the only "infinite" configuration of interacting spins is obtained when the spin interactions act along the same direction, i.e., the AFM Heisenberg chain, which is exactly soluble. ${ }^{22}$

In the next subsection, starting from an isolated tetrahedron we discuss possible orbital arrangements on the pyrochlore lattice and provide the solution of the corresponding spin Hamiltonian.

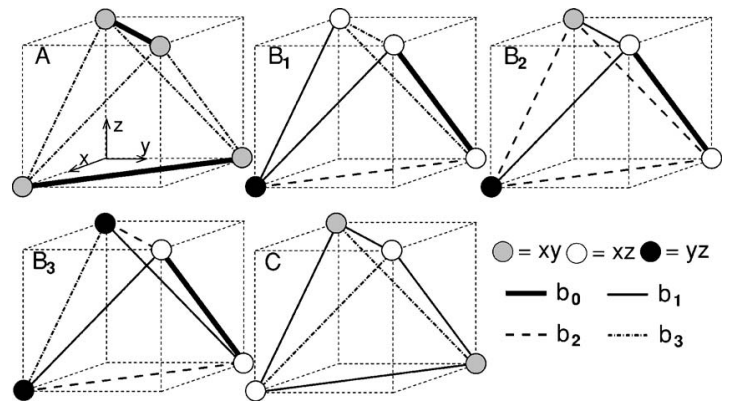

FIG. 2. The orbital and bond arrangements on a tetrahedron for cases $A, B_{1,2,3}$, and $C$ discussed in the text.

\section{B. Possible phases on a pyrochlore lattice and their energetics}

It is possible to show that only three energetically inequivalent tetrahedrons can be singled out of the four $b_{n}$ bonds introduced above. ${ }^{19}$ Topologically, they can be characterized by the number of strongest $b_{0}$ bonds and classified in three following types (pictorially shown in Fig. 2): A-type tetrahedra with two $b_{0}$ bonds, $B$-type tetrahedra with one $b_{0}$ bond, and $C$ tetrahedra with no $b_{0}$ bonds. Notice that, whatever the orbital configuration is, every single tetrahedron must obey the constraint $2 n_{b_{0}}+n_{b_{1}}=4$. This can be seen by introducing the operators that count the number of interacting $b_{0}$ and $b_{1}$ bonds on a tetrahedron. They are given, respectively, by $N_{b_{0}}^{\mathrm{T}}=\Sigma_{\langle i j\rangle}^{\prime} \widetilde{O}_{i j}$ and $N_{b_{1}}^{\mathrm{T}}=\Sigma_{\langle i j\rangle}^{\prime} O_{i j}$, with the summation restricted to one tetrahedron. Using the identity for projectors $P_{i, x y}+P_{i, x z}+P_{i, y z}=1$ (as there is one electron at each site), one obtains the operator identity $2 N_{b_{0}}^{\mathrm{T}}+N_{b_{1}}^{\mathrm{T}}=4$. This implies that $A$ tetrahedra have no $b_{1}$ bonds, $B$-type tetrahedra are characterized by $2 b_{1}$ bonds, and, for $C$ tetrahedra, there must be $4 b_{1}$ bonds. We can further classify $B$-type tetrahedra, according to the choice of the two orbitals on the tetrahedron bond opposite to the $b_{0}$ bond, into $B_{1}, B_{2}$, and $B_{3}$ (see Fig. 2) where, respectively, the bond opposite to the $b_{0}$ one is of $b_{1}, b_{2}$, and $b_{3}$ type. We stress again that the number of $b_{2}$ and $b_{3}$ bonds is not relevant as far as the Hamiltonian (1) is considered: they acquire importance only when magnetoelastic correlations among bonds will be introduced, in the next section, as they allow us to distinguish topologically the various configurations in the pyrochlore lattice. $A, B$, and $C$ tetrahedra are the bricks that allow us to build the orbital pattern throughout the whole pyrochlore lattice. Because of the Ising form of orbital interactions, in the following we can focus simply on these three cases, relying on the fact that configurations with a linear superposition of orbitals on each site must have a higher energy. We shall make only one exception to study a case with a particular physical meaning, i.e., that of a "cubic" symmetry, where each site is occupied by a linear superposition with equal weights of the three orbitals $1 / \sqrt{3}[|x y\rangle+|x z\rangle+|y z\rangle]$.

We can now cover the whole pyrochlore lattice with these tetrahedra: in principle, infinite coverings of the crystal are possible. Among them, we select the finite subclass that keeps the volume of the fcc cell. Thus the constraint is that any tetrahedron in the next cell be equivalent, even though in each cell they are no more equivalent to one another, as in 

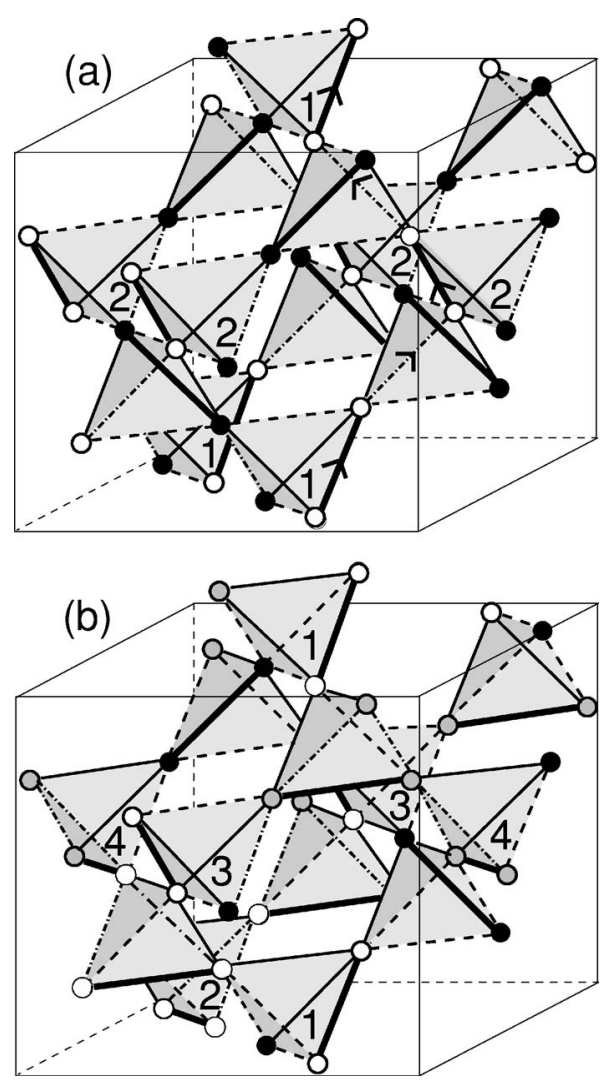

FIG. 3. The ground state coverings of the unit cubic cell through dimers. Locations of singlets are represented by thick links. Different numbers correspond to inequivalent tetrahedra. (a) The helical dimerization pattern (indicated by arrows) is formed by alternating short $b_{0}$ and long $b_{3}$ bonds, dimer phase $B_{3}$. (b) One of the possible coverings of the cubic unit cell by $B_{1} B_{2}$ tetrahedra.

Fig. 3(b). A posteriori, this choice is justified by the fact that the symmetry lowering characterizing the ground state [Fig. $3(\mathrm{a})$ ] is less than what is allowed by our constraint. We have the following global patterns:

(I) Heisenberg chains: If all tetrahedra of pyrochlore lattice are characterized by two $b_{0}$ bonds (i.e., all sites are occupied by the same orbital), then the effective Hamiltonian (1) can be mapped into a set of one-dimensional decoupled AFM Heisenberg chains that lie within the plane of the chosen orbital. The only interactions are due to $b_{0}$ bonds and are described by the spin Hamiltonian (3). Thus, the ground-state energy per site can be evaluated exactly by using the results for a Heisenberg chain, ${ }^{22}$ which give $E_{A}=-2.77(1-2 \eta) J$.

(II) Dimer phase $B$ : This state is made of only $B$-type tetrahedra with one strong $b_{0}$ bond and two intermediate $b_{1}$ bonds. As all three $B_{i}$ tetrahedra are energetically equivalent, all possible coverings of pyrochlore lattice by $B_{i}$ tetrahedra have the same energy. When the lattice is covered by $B_{i}$ tetrahedra then each spin can be engaged, in principle, in one or two strong AFM $b_{0}$ bonds. However, the coverings where each site belongs to just one $b_{0}$ bond are energetically more favorable, due to the full magnetic energy gain corresponding to the spin-singlet formation. In the other case the spins shared by the $b_{0}$ bonds do not allow us to achieve this maximum energy gain on each bond (note that the number of $b_{0}$ bonds is fixed in this phase and equal to the number of tetrahedra). Two possible coverings are shown in Fig. 3. As expected, each spin is also linked to two weak FM $b_{1}$ bonds. Such coverings form a degenerate manifold, and the corresponding energy can be calculated as follows. In the limit $\eta \rightarrow 0$, the spin-only Hamiltonian can be solved exactly, as it can be decomposed into a sum of spin-uncoupled $b_{0}$ bonds. In this case the energy minimum is reached when the Heisenberg term of the $b_{0}$ bond is the lowest, i.e., for a pure quantum spin singlet $\left(\vec{S}_{i} \cdot \vec{S}_{j}=-3 / 4\right)$. Remarkably, such spinsinglet (dimer) states, in the limit $\eta \rightarrow 0$, are also exact eigenstates of the full Hamiltonian (1). As $\eta \ll 1$, the dimer state is stable against the weak FM interdimer interaction. In this case the magnetic contribution along the FM $b_{1}$ bond is zero $\left(\left\langle\vec{S}_{i} \cdot \vec{S}_{j}\right\rangle=0\right.$ for $i$ and $j$ belonging to different dimers) and we are led to an energy per site given by $E_{B}=E_{b_{0}} / 2+E_{b_{1}}$ $=-\left(3-\frac{7}{2} \eta\right) J$. Here $E_{b_{0(1)}}$ is the energy of the bond $b_{0(1)}$.

(III) FM order: In this case all tetrahedra are of $C$ type with four interacting $b_{1}$ bonds and two $b_{3}$ (or one $b_{2}$ and one $b_{3}$ ) noninteracting bonds. Thus, all nonzero spin-exchanges are ferromagnetic and given by Eq. (4). The ground state for this type of orbital ordering is thus ferromagnetic and has an energy per site given by $E_{C}=2 E_{b_{1}}=-2(1+\eta) J$.

(IV) Frustrated AFM: Even if the "Ising" form of orbitals interaction implies that configurations with linear superposition of orbitals on each site must have a higher energy, we consider for completeness the case where each orbital is occupied by a linear superposition with equal weight of the three orbitals: $(1 / \sqrt{3})(|x y\rangle+|x z\rangle+|y z\rangle)$. The realization of this phase restores the full pyrochlore lattice symmetry and, after averaging Eq. (1) over the orbital configurations on neighboring sites $i$ and $j$, the system is described by the spin Heisenberg Hamiltonian $H_{D} / J=\Sigma_{i j}[-5 / 9+(4 / 9$ $\left.-16 \eta / 9) \vec{S}_{i} \cdot \vec{S}_{j}\right]$, whose ground-state energy per site is $E_{D}$ $\simeq-(1.89-0.89 \eta) J$. Here we borrowed the numerical evaluation for ground-state energy in a pyrochlore lattice from Ref. 23: $(1 / N) \sum_{i j} \vec{S}_{i} \cdot \vec{S}_{j} \simeq-0.5$. Such a state is highly frustrated and its ground state is a spin liquid. ${ }^{6}$ It seems worthwhile to note that the energy in this phase is higher than that of the other phases, as it does not exploit at all the potential energy gain contained in the orbital ordering. ${ }^{24}$

(V) Mixed (dimer) phase $A C$ : It is of course possible to cover the pyrochlore lattice also by means of mixed configurations of tetrahedra. There are, in principle, infinite possibilities in this sense, but those minimizing the energy must contain, on average, at least one singlet bond every tetrahedron, due to the big energy gain related to the spin-singlet state in $\mathrm{H}_{b_{0}}$, and two $b_{1}$ bonds. While it is not possible to have, on average, more than one spin-singlet bond every tetrahedron, ${ }^{25}$ one can fill the whole pyrochlore lattice by means of a mixed configuration made of alternated tetrahedra with two and zero $b_{0}$ bonds ( $A$ - and $C$-type tetrahedra, respectively). This configuration is shown in Fig. 4. For this type of orbital arrangement one can also construct an exact ground state of corresponding spin Hamiltonian, in the limit of vanishing Hund's coupling. The ground state is given by dimer phase where there are two spin singlets, located opposite each other, on strong $b_{0}$ bonds of $A$-type tetrahedra while 


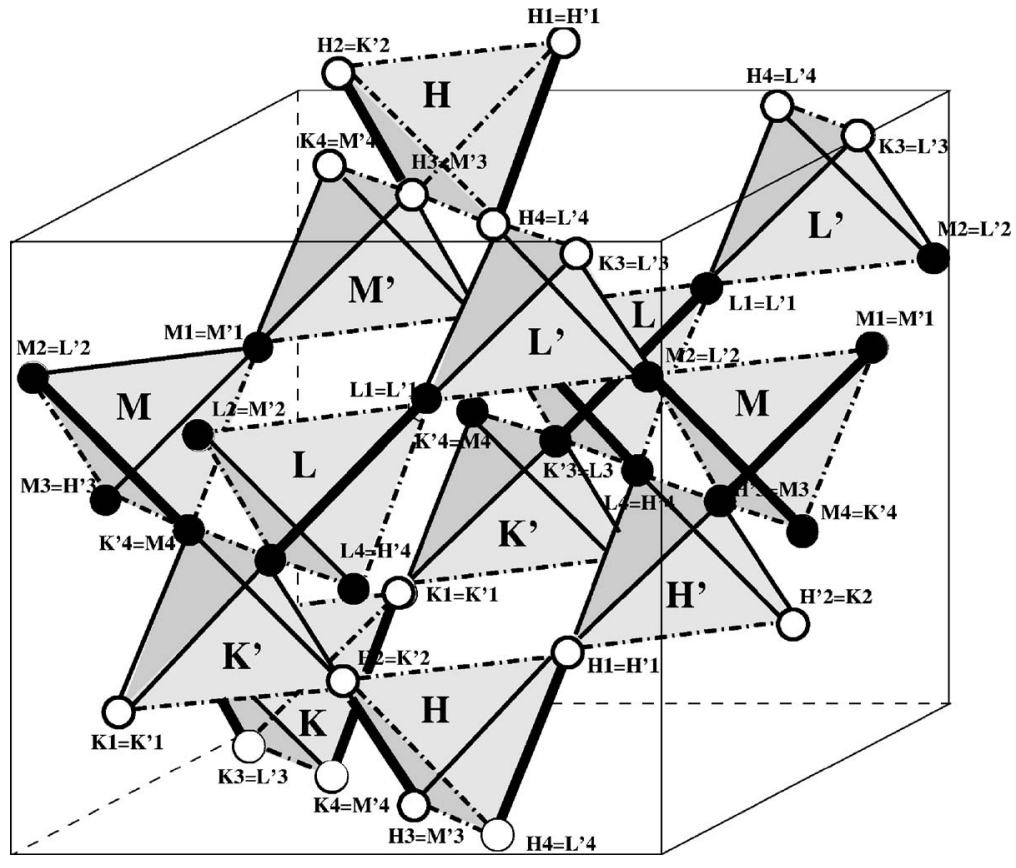

FIG. 4. The coverings of the unit cubic cell through the tetrahedra of $A$ type with two singlets and $C$ type with no singlets. Tetrahedra are labeled as in Fig. 6 .
$C$-type tetrahedra have no singlets. As on average, $n_{b_{0}}=1$ and $n_{b_{1}}=2$, this configuration is degenerate with dimer phase $B$, as far as only $d d \sigma$ overlap is considered.

\section{Ground state manifold}

Energies of the possible phases on the pyrochlore lattice can be discussed in terms of $\eta$, the only free parameter available. For $\eta=0$ the lowest ground-state energy is that of spinsinglet degenerate manifold. With increasing $\eta$ we find only one phase transition at $\eta_{c}=2 / 11 \simeq 0.18$, from spin-singlet manifold to a FM phase. As $\eta_{c}$ is above our estimated value of $\eta \simeq 0.15$, we can conclude that the ground state manifold is given by spin-singlet phases spanned by degenerate dimer states of $B$ or $A C$ type. This degenerate manifold is characterized by a static pattern of spin singlets (dimers) throughout the whole pyrochlore lattice and, thus, is different from the RVB state. Each dimer covering is frozen in an exact eigenstate of the Hamiltonian (1) for $\eta=0$. For finite $\eta$ the different dimer patterns are not connected by the Hamiltonian: the bond corresponding to the dimer in each tetrahedron is fixed, being determined by orbital pattern, and orbital degrees of freedom are static variables. Thus a tunneling between different dimer states cannot take place.

In this spin-singlet manifold the original spin degeneracy is removed. However, there is still a remaining degeneracy to be lifted. The degeneracy of the $B$ manifold is related to the freedom in the choice of the two orbitals on the tetrahedron bond opposite to the one of the singlet. Different choices of these orbitals give rise to inequivalent dimer covering patterns of the pyrochlore lattice with one dimer per tetrahedron (see Fig. 3). This degeneracy is given by the number of such dimer coverings and the corresponding number of states can be estimated as follows. In the $B$ manifold there is one singlet per tetrahedron and each spin can be engaged in only one singlet. Moreover, when a singlet is located on a given tetrahedron, then each neighboring tetrahedron is left with only three possible choices for a singlet location. Thus the number of such coverings grows with the system size as $\mathcal{N}_{B} \sim 3^{N_{T}}=\sqrt{3}^{N}$. Here $N_{T}=N / 2$ is the number of tetrahedra and we have ignored the contributions coming from closed loops (hexagons) on the pyrochlore lattice.

The asymptotical determination of $A C$-manifold degeneracy is a more difficult task. However, based on the simple arguments we can estimate its lower and upper limit correctly. First, we divide the pyrochlore structure in two sublattices formed by two differently oriented tetrahedra: one sublattice is composed by $A$-type tetrahedron with two singlets located opposite each other and the other by $C$-type tetrahedron with no singlets. Then consider an $A$ tetrahedron with two singlets in the $\alpha \beta$ plane: it can be directly verified that the four NN tetrahedra of the $A$ sublattice, connected to it by straight lines in the $\alpha \beta$ plane, will have only two possible orbital choices. The remaining eight NN tetrahedra of the $A$ sublattice have, instead, either two or three choices to locate the two spin singlets. These constraints are dictated by the condition that intermediate tetrahedron must be of $C$ kind. Thus, the degeneracy can be estimated as follows: $2^{N_{T}^{\prime}}<\mathcal{N}_{A C}<3^{N_{T}^{\prime}}$, where $N_{T}^{\prime}=N / 4$ is the number of tetrahedra in one sublattice. We conclude that the degeneracy of this manifold is still macroscopic, but smaller than that of the $B$ one.

Thus, even though the formation of spin-singlets removes the spin degeneracy of the pyrochlore lattice, there is still a macroscopic degeneracy to be lifted. The main question is whether this degeneracy can be removed by extending our effective Hamiltonian to the previously neglected $d d \pi$ and $d d \delta$ overlaps. When these processes are considered, $b_{2}$ and $b_{3}$ bonds acquire a different bonding energy, and, as the number of these bonds is not the same for $B_{i}$ and $A C$ manifolds, ${ }^{19}$ the degeneracy between these two phases can be lifted. However, this effect is smaller than the one induced by magnetoelastic coupling (see Sec. III), as $J_{d d \pi} \equiv t_{d d \pi}^{2} / U_{2}$ 


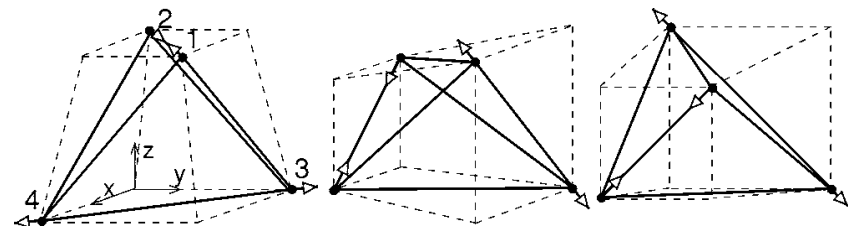

FIG. 5. The triplet-T deformation mode from the irreducible representations of the tetrahedron group. This mode generates a distortion of the tetrahedron, with short and long bonds located opposite to each other and four intermediate (undistorted) bonds.

$\simeq 2 \mathrm{meV}$, while magnetoelastic energy gain per ion is about $6.5 \mathrm{meV}$. It is then obvious to look for the degeneracy removal first in terms of this "spin-Teller" interaction, as done below.

Moreover, the degeneracy within the $B_{i}$ manifold cannot be removed within the effective electronic model, not even introducing smaller NN hopping integrals. The reason is related to the fact that the energy gain depends only on the total number of bonds of each type $\left(n_{b_{0}}, n_{b_{1}}, n_{b_{2}}, n_{b_{3}}\right)$ in the unit cell, and, in order to fill the whole crystal with a periodicity not lower than the one of the primitive cubic cell, the average number of bonds $n_{b_{i}}$ per tetrahedron should be the same, whichever of the three tetrahedral configurations is taken $\left(B_{1}\right.$, with $n_{b_{2}}=1$ and $n_{b_{3}}=2$; or $B_{2}$, with $n_{b_{2}}=3$ and $n_{b_{3}}=0$; or $B_{3}$, with $n_{b_{2}}=2$ and $n_{b_{3}}=1$ ). This number is given by $n_{b_{0}}=1, n_{b_{1}}=2, n_{b_{2}}=2, n_{b_{3}}=1$, and it corresponds to the value of the $B_{3}$ case, which is the only one that allows us to cover the whole cubic cell without mixing to other configurations (see Fig. 3).

From the above discussion it follows that only correlations between bonds can lift this degeneracy. These correlations naturally appear if the magneto-elastic contribution to the energy is considered. The orbitally driven modulations of the spin exchange interactions will distort the underlying lattice through the spin-Peierls mechanism and different distorted patterns will pay a different elastic energy. The three degenerate phases which will be discussed in the next section, are those with the unit cell filled by all $B_{3}$ tetrahedra (" $B_{3}$ phase"), that with a mixture of $B_{1}$ and $B_{2}$ tetrahedra (" $B_{1} B_{2}$ phase"), and that where the unit cell is filled by alternated $A$ and $C$ tetrahedra ("AC phase").

\section{EVALUATION OF THE MAGNETOELASTIC ENERGY}

In a spin-Peierls system the magnetic energy gain due to the spin-singlet pairs outweighs the increase in elastic energy due to the dimerization of the regular array. We have shown with qualitative arguments ${ }^{19}$ that in our case this mechanism can select the triplet- $T$ normal mode of the tetrahedron group, leading to a distortion with one short and one long bond located at opposite edges and four undistorted bond (see Fig. 5). Indeed, a reduction of the bond length increases the magnetic energy gain and therefore favors the shortening of the bond with the strongest superexchange, i.e., $b_{0}$, where the singlet is located.

Even though this picture is correct in its basic features, a quantitative description of the global physical mechanism leading to the tetragonal distortion requires a more careful analysis, which takes into account all elastic normal modes of the single tetrahedron, as well as the correlations of these normal modes within the unit cell. The aim of the present section is to analyze just such a global mechanism.

The dependence of the energy gain $\Delta E$ on the magnitude of distortion can be evaluated as a sum of magnetic $\Delta E^{m}$ and elastic $\Delta E^{e l}$ contributions at each bond:

$$
\Delta E=\sum_{i j} \Delta E_{i j}^{m}+\Delta E_{i j}^{e l}
$$

The sum is restricted to the NN sites.

All bonds in the undistorted lattice have the same length, and we represent their elastic energy assuming that all ions are connected to one another through equal springs of constant $k$ :

$$
\Delta E_{i j}^{e l}=\frac{1}{2} k\left(\delta d_{i j}\right)^{2}=\frac{1}{2} C_{0} \frac{\left(\delta d_{i j}\right)^{2}}{d_{0}^{2}},
$$

where the constant $C_{0} \equiv k d_{0}^{2}$ is the radial force constant ${ }^{26}$ and $\delta d_{i j} \equiv d_{i j}-d_{0}$ is the deviation of the bond length from its value $d_{0}$ in the undistorted cubic lattice.

The magnetoelastic energy of the generic bond $i j$ can be written as

$$
\Delta E_{i j}^{m}=\left[J\left(d_{0}\right)+g \delta d_{i j}\right] \vec{S}_{i} \cdot \vec{S}_{j}
$$

where $\left.g \equiv[\partial J(d) / \partial d]\right|_{d=d_{0}}$. The dependence of exchange constants on the distance is mediated by the hopping matrix element: exchange constants are proportional to $t^{2}$, and $t$ is inversely proportional to the fifth power of the distance. In Ref. 26 it is possible to find a rough estimate of the proportionality constant, $\alpha: t=\alpha / d^{5}$, with $\alpha=\frac{3}{4} \eta_{d d \sigma}\left(\hbar^{2} r_{d}^{3} / m\right)$. Here $r_{d}$ is a characteristic length, that for $\mathrm{Ti}$ is $1.08 \AA$, while $\eta_{d d \sigma}=-16.2$ and $\hbar^{2} / m=7.62 \mathrm{eV} \AA{ }^{2}$ giving the value $\alpha=-116.628 \mathrm{eV} \AA .^{5}$ It is immediately apparent that even a small reduction in the length of a given bond can lead to a relatively high magnetic-energy gain.

In order to analyze the magnetoelastic behavior of the three degenerate phases of our spin-orbital model we first focus on a single tetrahedron, either of $A, B_{i}$, or $C$ type. For a more fluent reading, the detailed calculations are reported in Appendix B, and here we just comment on the main points.

In general, the global magnetoelastic Hamiltonian involves more than one normal mode for each tetrahedron: for example, for $A$ tetrahedra the singlet and one doublet mode contribute to the magnetoelastic energy, and for $B_{2}$ tetrahedra all six normal modes are involved, as reported in Appendix B. Moreover, even though the triplet $T$-mode associated the singlet bond leads to a bigger energy gain for $B_{3}$ than for $B_{1}$ tetrahedra, when all available normal modes are considered, the total energy gain gets different contributions from all normal modes, but in such a way that it is the same for all three $B_{i}$ tetrahedra, and equal to $-4.5 g^{2} / k$. What makes the difference among these configurations in a specific case like that of $\mathrm{MgTi}_{2} \mathrm{O}_{4}$ is that when these tetrahedra are considered in an infinite lattice, the single-tetrahedron degeneracy is lifted by the correlations among tetrahedra. In fact, in corre- 


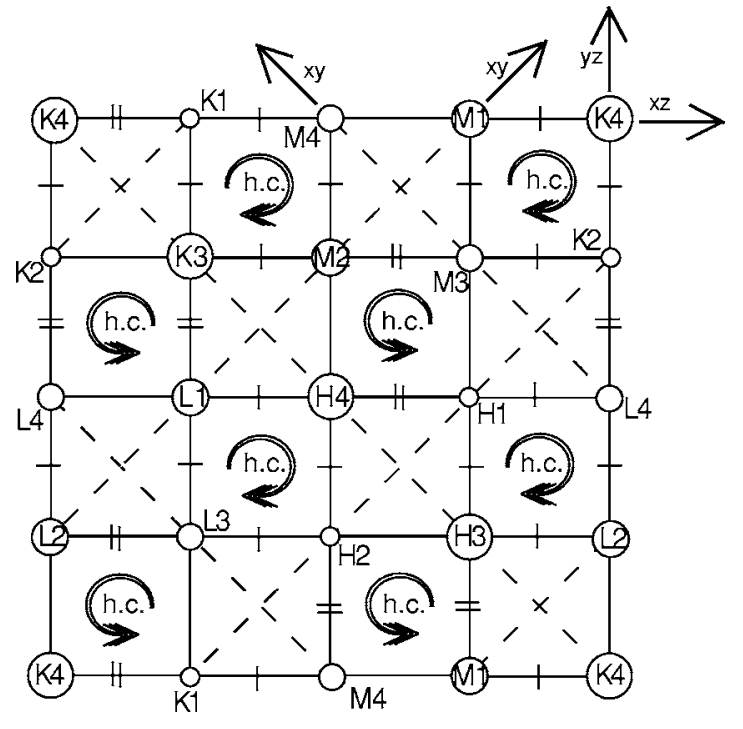

FIG. 6. The bidimensional projection of the unit cell. Circles represent Ti ions, with the same labels as in Fig. 4. Diameters are proportional to the $z$ coordinate of the ion: $z=1,0.75,0.5,0.25$, in fractional units. All bonds are oriented along one of $x y, x z, y z$ bisectors, as indicated. Helical chains (h.c.) are represented in the direction of increasing $z$. Doubly cut bonds are $b_{0}$ bonds and singly cut are $b_{1}$ bonds.

spondence with their common energy minimum, $B_{3}, B_{1}$, and $B_{2}$ tetrahedra are all distorted differently, as can be deduced from the location of their minima. Thus, when these distortions are combined together in order to fill in exactly the global cubic cell, as in Fig. 6, the appearance of new constraints, due to the relative match of all tetrahedra within the cell, forces the solution to another minimum. For example, in order to keep the global volume unaltered, all bond distortions due to spin singlets must appear with reversed sign from one tetrahedron to the other, because to each expansion there must be a corresponding contraction. Notice that also the $A C$ phase is still degenerate with $B_{i}$ configurations, as far as just magnetoelastic energy gain associated with single tetrahedra is considered, as, on average, it is again $(-8-1) / 2 g^{2} / k=-4.5 g^{2} / k$ (see Appendix B). Remarkably, when intertetrahedra correlations are considered, elastic singlet modes contribute to $A C$ phase differently than $B_{i}$ phase. In fact, it is worthwhile for the system to expand $C$ tetrahedra and contract $A$ tetrahedra of an equal amount, as this leads to a net energy gain, because the singlet mode energy gain of $A$ tetrahedra exceeds that of $C$ ones.

It is clear from these considerations that in order to get the global magnetoelastic minimum for the unit cubic cell we cannot rely on the unconstrained minimization of the single tetrahedra shown in Appendix B. One alternative possibility is to calculate the magnetoelastic energy for the normal modes of the global cubic cell for the three degenerate configurations $\left(B_{3}, B_{1} B_{2}\right.$, and $A C$-phase), in such a way as to automatically consider all constraints among tetrahedra. Yet this procedure is not complete, as normal modes corresponding, e.g., to the buckling of tetrahedra do not contribute to the magnetoelastic energy. Such a contribution appears only when the normal mode involves an increase/decrease of some bond length, and, thus, it can be expressed as a linear combination of the normal modes of each single tetrahedron, with the constraint that the position of each equivalent $\mathrm{Ti}$ ion over all nearest-neighbor unit cells be the same $(\mathbf{k}=0$ modes).

In order to fulfill this constraint, we impose that the total length over all the straight bonds connecting one ion with the equivalent ones in the next cubic cells is a conserved quantity. This means that, for example, $d_{H 4-H 3}+d_{H 3-K 4}+d_{K 4-K 3}$ $+d_{K 3-H 4}$ (see Fig. 6) is a conserved quantity, and, thus, the sum of the four displacements $\delta d_{H 4-H 3}+\delta d_{H 3-K 4}+\delta d_{K 4-K 3}$ $+\delta d_{K 3-H 4}=0$. The same happens for the other three chains in the $x y$ plane, for the four of the $x z$ plane, and for the four in the $y z$ plane. Due to Eqs. (B4), these 12 conditions become constraints for the normal variables of the different tetrahedra. The search for the minima of the magnetoelastic Hamiltonians (B5)-(B9) with the previous constraints leads to the following expression of the energy per unit cell: $-18 g^{2} / k$ for $B_{3}$ phase, $-17.2 g^{2} / k$ for $B_{1} B_{2}$ phase, and $-14.4 g^{2} / k$ for $A C$ phase. Thus, the ground state is given by $B_{3}$, with an energy per tetrahedron of $-2.25 \mathrm{~g}^{2} / \mathrm{k}$, corresponding to the sum of the energies of the triplet $t_{2}$ and doublet $e_{1}$ modes. The singlet and the doublet $e_{2}$ modes do not contribute to the energy, when they are constrained on the global unit cell. As now each ion is shared by two tetrahedra, the energy per site is $-1.125 g^{2} / k$.

However, there is an experimental fact that is not properly explained by this solution, and for which an extra discussion is required. The solution of the constrained minimization gives an amplitude of $2 \mathrm{~g} / \mathrm{k}$ for triplet $t_{2}$ mode and $\mathrm{g} / \mathrm{k}$ for doublet $e_{1}$ mode, thus a ratio 2:1 in favor of the triplet mode. But the experimentally reported tetrahedral distortions ${ }^{10}$ are, referring to Eqs. (B3) and (B4), $\delta d_{13}=-0.155 \AA$, and $\delta d_{14}$ $=-0.006 \AA$, which imply, when only $t_{2}$ and $e_{1}$ normal modes are active, $\theta_{e 1}=-0.012 \AA$ and $\theta_{t 2}=-0.149 \AA$, with a tripletto-doublet ratio of more than 10 . This indicates that, in the previously outlined model, some features of the real system are not taken into account, like the elastic potential coming from the oxygens, which acts in such a way as to reduce some distortions. Thus, the possibility to get better quantitative results for the experimental bond lengths within our scheme should pass through a way to mimic this aspect by means of a stronger constraint on the available modes before the minimization procedure. If we consider Fig. 6, we can see that the ion $\mathrm{H} 4$ can be connected to the equivalent ones in the neighboring edge-sharing unit cells through one of the three "linear" paths: $H 4-H 1-L 4-L 1-H 4$ in the $x z$ plane, or $H 4-H 2-M 4-M 2-H 4$ in the $y z$-plane, or $H 4-H 3-K 4-K 3-H 4$ in the $x y$ plane. Face-sharing unit cells are connected by the paths along the helical chains, namely, H4-H2-L3-L1-H4 and $H 4-H 1-M 3-M 2-H 4$ for chains along the $z$ axis, $H 4-$ $H 2-K 1-K 3-H 4$ and H4-M2-M1-H3-H4 for chains along the $x$ axis, and H4-H1-K2-K3-H4 and H4-H3-L2-L1-H4 for chains along the $y$ axis. In principle the global $H 4-H 4$ distance along, e.g., the $z$ axis can be kept fixed even if the sum of the four bonds changes, as the bonds are not along a straight line, and the elongation of one bond can be combined with an appropriate rotation, due to a tetrahedron buckling. Yet, this buckling implies an extra elastic energy loss due to the relative movement with $\mathrm{Mg}$ and $\mathrm{O}$ ions. We 
can forbid these extra losses by imposing another series of constraints that express the global conservation of the total length also along the "helical" chains, in this way freezing some of the normal modes that were previously allowed.

When the minimization is performed with this new constraint, the results for the energy per unit cell are $-16 g^{2} / k$ for $B_{3}$ phase, $-13 g^{2} / k$ for $B_{1} B_{2}$ phase, and $-g^{2} / 3 k$ for $A C$ phase. Again, $B_{3}$ phase is the lowest, but this time the amplitude of $e_{1}$ mode is zero and the minimum energy is reached for a pure triplet mode, of amplitude $2 g / k$. Notice that with this constraint the $A C$ phase gains very few, and also the relative energy difference between $B_{1} B_{2}$ phase and $B_{3}$ increases.

Finally, let us stress again that the main result is that when correlations among magnetoelastic energy are taken into account, $B_{3}$ phase is always stabilized with respect to the two configurationally degenerate $B_{1} B_{2}$ and $A C$ ones. Of the two criteria used for preserving the cell volume, the second seems more in keeping with experimental data. This suggests that oxygens play a role in the elastic distortions of the spinel cell, which is more rigid towards bucklings than the bare pyrochlore lattice. In any case it is found that the elongation of the non-interacting $b_{3}$ bond and the corresponding stretching of $b_{0}$ bond throughout the whole cell is always energetically more favorable, as found experimentally.

Given the previous $d$ dependence of the hopping amplitude $t$, it is possible to estimate the gain energy per ion associated with this magnetoelastic distortion. First, $g$ $\equiv-\left.(\partial J / \partial d)\right|_{d=d_{0}}=10 J / d_{0}$. Using Eq. (B4), $\delta d_{13}=-0.155 \AA$ $=-2 \mathrm{~g} / \mathrm{k}$, with $k=C_{0} / d_{0}^{2}$. This implies that $C_{0}=10 \mathrm{~J} d_{0} / 0.155$ $\simeq 9.7 \mathrm{eV}$, if we take $d_{0} \simeq 3.008 \AA$. Finally, the estimate of the average magnetoelastic energy per site can be easily found: there are 16 ions in one unit cell, and thus the energy per site is $E_{m e}=-g^{2} / k=(10 J)^{2} / C_{0} \simeq 6.5 \mathrm{meV}$.

\section{EFFECT OF MAGNETIC FIELD}

As we have shown above, the ground state of the system is nonmagnetic and spins are paired in singlet states. The spin degrees of freedom are thus gapped and the gap of triplet excitations is given by the singlet binding energy $\Delta_{s}$ $=4 J(1-2 \eta)[$ see Eq. (3) $]$. When an external magnetic field is applied, the energy of triplet excitations decreases, due to the gain of Zeeman energy, while the singlet state does not experience the applied field. Therefore, one would expect no change in the symmetry of the ground state up to fields $g \mu_{B} H_{c}=\Delta_{s}$ at which singlet triplet gap closes ( $g$ is the gyrotropic factor and $\mu_{B}$ is the Bohr magneton). At $H=H_{c}$ a second order transition can, in principle, take place from nonmagnetic to a magnetically ordered state, driven by condensation of lowest energy triplets at some ordering wave vector $\mathbf{Q}$ at which triplet spectrum has a minimum.

However, the presence of an orbital degeneracy can modify this convectional picture. As shown in Sec. II, the first excited state above dimer phase is the FM one, characterized by $C$ orbital pattern. The energy of FM phase, contrary to the dimer phase, is sensitive to the applied magnetic field and decreases of $\Delta E_{H}=-g H \mu_{B} S_{i}^{z}$. The critical field $\widetilde{H}_{c}$ at which the energies of the two phases are equal is

$$
g \mu_{B} \tilde{H}_{c}=J(2-11 \eta)
$$

and one can easily verify that $\widetilde{H}_{c}<H_{c}$. Therefore at $H=\widetilde{H}_{c}$ there will be a first-order transition from dimer phase to FM state. This transition will be accompanied by a simultaneous rearrangement of orbital ordering and the abrupt closure of the spin gap, as the FM phase is gapless. We can estimate the order of magnitude of $\tilde{H}_{c}$, for $\eta \simeq 0.15$ and $J=25 \mathrm{meV}$, as $\mu_{B} \tilde{H}_{c} \simeq 4.4 \mathrm{meV}$, i.e., a critical field of $\tilde{H}_{c} \simeq 76 \mathrm{~T}$. In this estimate we did not consider the extra stabilization energy in favor of the singlet phase coming from the magnetoelastic distortion. Of course, one can read Eq. (8) the other way round, in terms of $\eta$, and deduce that, if a critical field is found at a lower value than $76 \mathrm{~T}$, this implies that $J_{H} / U_{2}$ is closer to the critical value of 0.18 than what was estimated here.

\section{DETECTION OF ORBITAL ORDERING THROUGH RXS}

\section{A. General remarks}

As already outlined in the previous two sections, orbital ordering in $\mathrm{MgTi}_{2} \mathrm{O}_{4}$ is mainly dictated by superexchange interactions. This implies that the orbital orientation at each site does not strictly follow the symmetry imposed by the local crystal field, as happens when the effect is purely determined by Jahn-Teller distortions. Therefore, we believe that there is the possibility to exploit the local symmetry differences in the helical $d_{x z}-d_{y z}$ orbital pattern by means of resonant $\mathrm{x}$-ray scattering (RXS), where the local transition amplitudes are added with a phase factor that can compensate the vanishing effect due to the global tetragonal symmetry.

Conceptually, this procedure is the analog of the one used in the case of manganites ${ }^{27}$ that led to a series of results initially interpreted as a direct evidence of orbital ordering, ${ }^{28}$ and soon later recognized ${ }^{13-15}$ as mainly determined by the oxygen distortion around each $\mathrm{Mn}$ ion. In this case the signal depended on the difference between, e.g., $4 p_{x}$ and $4 p_{y}$ projected density of states at the resonant energy. ${ }^{29}$ Such local anisotropies in the electronic $4 p$ density of states can be a consequence of oxygen distortions (Jahn-Teller effect) or can be induced by the ordering of the underlying $3 d$ orbitals through the $3 d-4 p$ Coulomb repulsion $U_{d p}$ (orbital ordering effect). ${ }^{14}$ The results of Refs. 13-15 independently show that the effect of the Jahn-Teller mechanism is much stronger, about a factor of 10 in amplitude, than the effect of orbital ordering. This led to the conclusion that $\mathrm{OO}$ could not be "directly" probed by means of RXS at K edges, where the strength of the ligand field overwhelms the effect induced by $U_{d p}$.

Due to this result, the search for experimental evidence of orbital ordering moved to L edge $\mathrm{RXS}^{30}$ where one is sensitive directly to $3 d$ orbitals. Yet, this kind of spectroscopy has the serious drawback that, in order to have nonimaginary Bragg angles, it is necessarily limited to crystals with very big unit cells, and this is not feasible for $\mathrm{MgTi}_{2} \mathrm{O}_{4}$. In fact, $\mathrm{Ti}$ $\mathrm{L}_{2,3}$ edges are at about 453.8 and $460.2 \mathrm{eV}$, which corre- 
sponds to a photon wavelength $\lambda \simeq 27 \AA$, which does not allow Bragg law $\sin \theta_{B}=\lambda / 2 d$ to be verified, not even for (001) reflection.

In spite of all this, we believe that it is still possible to detect the proposed $\mathrm{OO}$ at $\mathrm{K}$ edge. The key point to go beyond the conclusions of Refs. 13-15 lies in the fact that their results are strongly related to the ratio between $U_{d p}$ and Jahn-Teller distortion. In particular, while the signal induced by the Coulomb repulsion $U_{d p}$ is of the order of $\simeq 200-300 \mathrm{meV}$, independently of the particular crystal structure, ${ }^{31}$ the influence of the oxygen distortion is much lower in the directions of $t_{2 g}$ orbitals than in $e_{g}$ ones. It might indeed seem conceivable that a reduced Jahn-Teller distortion can give rise to a sizable interference with the OOinduced effect in the RXS intensity. Moreover, there is the possibility that the rotation of the exchange vector and/or polarizations in the RXS experiment can make the effect more or less pronounced, according to the experimental conditions. We have thus performed a numerical simulation and found that it is indeed possible to experimentally reveal the presence of the OO through the comparison of different RXS signals. In the following we illustrate the details of our calculations, in order to clarify the previous theoretical speculations.

\section{B. Derivation of the structure factor}

The transition process for RXS, governed by the Fermi golden rule, depends on the state overlap through the polarized electric field of the incoming $(i)$ or outgoing $(o)$ photon. If we consider the multipole expansion up to the electric quadrupole contribution, we get

$$
M_{n g}^{i(o)}=\left\langle\psi_{n}\left|\vec{\epsilon}^{i(o)} \cdot \vec{r}\left(1-\frac{1}{2} i \vec{k}^{i(o)} \cdot \vec{r}\right)\right| \psi_{g}\right\rangle .
$$

Here $\psi_{g}$ and $\psi_{n}$ are ground and intermediate state wave functions, respectively, $\vec{\epsilon}^{i(o)}$ is the polarization of the incoming (outgoing) photon, and $\vec{k}^{i(o)}$ is its wave vector. Around an absorption edge, $M_{n g}$ is highly energy and angular dependent and this provides the sensitivity to the electronic structure around the atom. In RXS the global process of photon absorption, virtual emission of the photoelectron, and subsequent decay with reemission of a photon is coherent, thus giving rise to the usual Bragg diffraction condition. The outgoing photon can have different polarization and wave vector compared to the incoming one and the atomic anomalous scattering factor (ASF) reads ${ }^{32}$

$$
f=f^{\prime}+i f^{\prime \prime}=\frac{m_{e}}{\hbar^{2}} \frac{1}{\hbar \omega} \sum_{n g} \frac{\left(E_{n}-E_{g}\right)^{3} M_{n g}^{o^{*}} M_{n g}^{i}}{\hbar \omega-\left(E_{n}-E_{g}\right)-i \frac{\Gamma_{n}}{2}} .
$$

Here $\hbar \omega$ is the photon energy, $m_{e}$ is the electron mass, $E_{g}$ and $E_{n}$ are the ground and intermediate state energies, and $\Gamma_{n}$ is the broadening of the transition. The sum over the intermediate states starts from the Fermi energy. ${ }^{18}$

In the tetragonal phase of $\mathrm{MgTi}_{2} \mathrm{O}_{4}$ the space group is the chiral group $\mathrm{P}_{1} 2_{1} 2$, No. 92 of Ref. 33 (or its "mirror"related $\mathrm{P} 4_{3} 22_{1}$, No. 96), with eight ions per unit cell, and the structure factor is
TABLE I. The orbital occupancy and tetragonal position of Ti ions. Symmetry operations are referred to $\mathrm{Ti}_{1}$.

\begin{tabular}{cccc}
\hline \hline Atom & OO & Position & Symmetry \\
\hline $\mathrm{Ti}_{1}(H 3)$ & $d_{y z}$ & $(u, v, w)$ & $\hat{E}$ \\
$\mathrm{Ti}_{2}(L 4)$ & $d_{y z}$ & $(-u,-v, 1 / 2+w)$ & $\hat{C}_{2 z}$ \\
$\mathrm{Ti}_{3}(H 1)$ & $d_{x z}$ & $(1 / 2-v, 1 / 2+u, 1 / 4+w)$ & $\hat{C}_{4 z}^{+}$ \\
$\mathrm{Ti}_{4}(L 2)$ & $d_{x z}$ & $(1 / 2+v, 1 / 2-u, 3 / 4+w)$ & $\hat{C}_{4 z}^{-}$ \\
$\mathrm{Ti}_{5}(L 3)$ & $d_{x z}$ & $(1 / 2-u, 1 / 2+v, 1 / 4-w)$ & $\hat{C}_{2 y}$ \\
$\mathrm{Ti}_{6}(H 4)$ & $d_{x z}$ & $(1 / 2+u, 1 / 2-v, 3 / 4-w)$ & $\hat{C}_{2 x}$ \\
$\mathrm{Ti}_{7}(H 2)$ & $d_{y z}$ & $(v, u,-w)$ & $\hat{C}_{2 x x}$ \\
$\mathrm{Ti}_{8}(L 1)$ & $d_{y z}$ & $(-v,-u, 1 / 2-w)$ & $\hat{C}_{2 \bar{x} x}$ \\
\hline \hline
\end{tabular}

$$
A(\vec{Q})=\sum_{j=1}^{8} e^{i \vec{Q} \cdot \vec{R}_{j}} f_{j}
$$

where $f_{j}$ is the ASF of ion $j$. The atomic positions, their orbital filling, and the symmetries relating the ions to one another are schematized in Table I. Notice that the tetragonal cell is characterized by a $45^{\circ}$ rotation in the $x y$ plane, compared to the cubic cell of Fig. 2(a).

Here $u=0.9911, v=0.2499$, and $w=0.8668$ are the fractional coordinates of the atoms in unit of tetragonal axes.

In the following we analyze all reflections $(00 l)$, which are Bragg forbidden unless $l=4 n$. Using the symmetries of Table I, the structure factor can be expressed in the following form:

$$
A(00 l)=\left[1+(-)^{l} \hat{C}_{2 z}\right]\left[1+(i)^{l} \hat{C}_{4 z}^{+}\right]\left[e^{2 \pi i l w}+(i)^{l} e^{-2 \pi i l w} \hat{C}_{2 y}\right] f_{1} .
$$

Note that even if $f_{1}$ is a scalar, it is expressed as a scalar product of two tensors, and, in Eq. (12), rotation operators are understood as applied to one of them. We can limit to the dipole approximation, where we just need to consider rank-2 Cartesian tensors, $T_{\alpha \beta}$, whose irreducible representation in $\mathrm{SO}(3)$ are a scalar, a pseudovector, and a traceless symmetric tensor: the first never contributes to Bragg-forbidden reflections (which are scalar forbidden), while the second is proportional to a magnetic moment and has no influence on nonmagnetic systems like $\mathrm{MgTi}_{2} \mathrm{O}_{4}$. The third tensor has five independent components, which can be labeled according to the usual second-rank real spherical harmonics as $D_{x y}, D_{x z}$, $D_{y z}, D_{x^{2}-y^{2}}$, and $D_{3 z^{2}-r^{2}}$. They are a measure of the anisotropy of $p$ density of states projected on the resonant ion. ${ }^{34}$ In order to determine the implications of Eq. (12) on the various reflections, we need to know how the symmetry operators act on our symmetric tensors. The indices of each tensor change according to the following rules: $\hat{C}_{2 z}(x, y, z)=(-x,-y, z)$, $\hat{C}_{2 y}(x, y, z)=(-x, y,-z)$, and $\hat{C}_{4 z}^{+}(x, y, z)=(y,-x, z)$. Similar results are obtained reminding us that the ASF in our approximation is a second-rank irreducible tensor $f_{m}^{(2)}$, and $\hat{C}_{2 z} f_{m}^{(2)}=(-)^{m} f_{m}^{(2)}, \hat{C}_{4 z}^{+} f_{m}^{(2)}=i^{m} f_{m}^{(2)}$, and $\hat{C}_{2 y} f_{m}^{(2)}=(-)^{m} f_{-m}^{(2)}$. As a consequence of Eq. (12) we get that all Bragg-forbidden re- 
flections of the kind $(0,0,4 n+1)$ and $(0,0,4 n+3)$ are sensitive to the complex mixture of density of states: $D_{x z} \pm i D_{y z}$ $\propto Y_{ \pm 1}^{2}$. For this reason, due to the cylindrical symmetry, a constant azimuthal scan is expected as well as a minor dependence on the orbital anisotropies of the $x z$ and $y z$ kind. On the contrary, Bragg-forbidden reflections of the kind $(0,0,4 n+2)$, if we neglect $\cos 4 \pi w \simeq 0.1 \ll \sin 4 \pi w \simeq 1$, are just proportional to $D_{x y}$ : thus, they must show a nonconstant azimuthal scan, and, indirectly, manifest some properties related to the $\mathrm{OO}$, through the depletion of the filled $d_{x y}$ orbitals.

If we express the polarization dependence in the various channels, referring to the cubic frame of Fig. 1, we get the following results. Both (001) and (003) reflections couple to $\epsilon_{x} \epsilon_{z}$ and $\epsilon_{y} \epsilon_{z}$, and thus they are different from zero only in the $\sigma \pi$ channel. As their amplitude is proportional to $(\sin \phi+i \cos \phi)$, they have a constant azimuthal scan. Instead, (002) reflection is detectable in all $\sigma \sigma, \sigma \pi$, and $\pi \pi$ channels, with an intensity that scales, respectively, as $\sin ^{2}(2 \phi), \sin ^{2} \theta_{B} \cos ^{2}(2 \phi)$, and $\sin ^{4} \theta_{B} \sin ^{2}(2 \phi)$, where $\theta_{B}$ is the Bragg angle. As $\sin ^{2} \theta_{B} \simeq 0.1$, signals in the $\pi \pi$ channel are $1 / 10$ smaller than those in $\sigma \pi$ and $1 / 100$ smaller than those in the $\sigma \sigma$ channel. Also their azimuthal scans are out of phase: that of the $\sigma \pi$ channel has its maximum value when $\phi=0$, i.e., in the direction of the nearest-neighbor oxygens, while those of the $\sigma \sigma$ and $\pi \pi$ channels take their maximum value along the $\mathrm{Ti}-\mathrm{Ti}$ chains in the $x y$ plane, where the $\sigma \pi$ signal is zero.

\section{Results}

In Fig. 7 we show the results of our numerical simulations for $(00 l)$ reflections, performed with the finite difference method option of the FDMNES program. ${ }^{18}$ As an input file we used the refined positions of Ref. 10 for $\mathrm{Mg}$, Ti, and $\mathrm{O}$ ions. Because of the increasing CPU-time consumption, we performed our simulation, with and without orbital ordering, just for a cluster of radius $2.5 \AA$, and one of radius $3.3 \AA$, the first including the oxygen octahedron and the second including also all six nearest neighbors Ti ions around the resonant atom. All assertions stated before, regarding the relative intensities and polarization dependence, have been confirmed numerically. Due to the chiral nature of the $\mathrm{P}_{4}{ }_{1}{ }_{1} 2$ space group, we have checked the possibility to detect a signal through x-ray natural circular dichroism. Unfortunately, the dichroic signal is too low (less than $0.05 \%$ of the absorption) and the changes induced by $\mathrm{OO}$ are practically negligible.

The main result of our numerical simulations lies in the comparison of Figs. 7(a) and 7(b), which shows how to reveal the presence of $\mathrm{OO}$ through a simple experiment. In fact, the $\mathrm{OO}$ case is characterized by a big increase in intensity of (002) reflection, of a factor of 1.7 , while (003) is left unaltered and (001) increases by no more than 1.3. Thus, an experimental setup that measures these signals keeping track of the relative intensity is sufficient to determine whether the orbital order is present or not: if the three reflections have almost the same intensity, there is no OO, while if the (002) is about twice that of the others, this is the signature of the $\mathrm{OO}$ of the kind predicted by our theory.
In order to understand the reasons behind this enhancement in intensity, it is useful to study $\mathrm{K}$ edge absorption, with and without $\mathrm{OO}$, in the fictitious system where the only absorption center is $\mathrm{Ti}_{1}$ ion. In fact, in this way we probe directly the $p$ density of states projected on $\mathrm{Ti}_{1}$ ion. Thus, linear dichroism, e.g., along (100) and (010) tetragonal directions, is sensitive to what we called $D_{x y}$. In Fig. 8 we report the absorption for a single $\mathrm{Ti}$ ion surrounded by the oxygen octahedron. Oxygens in the $x y$ plane almost lie along cubic $x$ and $y$ axes, slightly squeezed towards the tetragonal $x^{\prime}$ axis. Labels in Fig. 8 refer to the tetragonal system, so that (100) direction corresponds to the incoming polarization along the line connecting the nearest-neighbor $\mathrm{Ti}$ ions in the $x y$ plane ( $45^{\circ}$ from the $x$ axis of Fig. 2). It is evident that in all figures, except Fig. 8(a), the presence of OO does not change the signal and the dichroism is entirely due to the ligand distortion. In particular, Fig. 8(c) shows the dichroism in the $y z$ cubic plane, while Fig. 8(d) is taken in the $x z$ plane and Fig. $8(\mathrm{~b})$ is a measure of $p_{x}-p_{y}$, towards the oxygens. On the contrary, Fig. 8(a) shows, in correspondence to its flex, an offset between the two curves varying from about $0.6 \mathrm{eV}$, in the absence of $\mathrm{OO}$, to $0.9 \mathrm{eV}$, when $\mathrm{OO}$ is present. Thus, along this direction $\mathrm{OO}$ can influence the RXS signal by a non-negligible amount, as shown in Fig. 7.

Finally, we want to comment about the influence of the cluster radius on the results. A series of numerical simulations of the local absorption (like those of Fig. 8) has been performed with radii $2.5,3.3$, and $4.0 \AA$, by means of the muffin tin program. The results show that the maximum dichroism between the (100) and (010) absorptions due to Jahn-Teller corresponds to the 3.3 radius, where, compared to the 2.5 cluster, two Ti ions are added along the (100) tetragonal direction, whereas no Ti ions are present along the (010). The simulations with the $4.0 \AA$ cluster show again a reduction of dichroism compared to $3.3 \AA$, due to the cell composition. Thus, the results shown in Fig. 7 for the cluster radius of $3.3 \AA$ correspond to the theoretical lowest ratio between the OO signal and JT one, and, thus, the effect of $\mathrm{OO}$ is expected to be not lower than that shown in Fig. 7(b).

\section{CONCLUSIONS}

To conclude, we have derived a mechanism that allows us to lift the geometrical degeneracy in a pyrochlore lattice of threefold orbitally degenerate $t_{2 g}$, spin-1/2, magnetic ions like $\mathrm{Ti}^{3}$. We have singled out two relevant energy scales that govern its behavior: the main one is determined by superexchange interactions, which drive the system into a spinsinglet dimer state with peculiar orbital pattern. The residual orientational degeneracy is then lifted through the magnetoelastic coupling, which optimizes the magnetic energy gain through a global tetragonal distortion of the system. This can be seen as a collection of $1 \mathrm{D}$ helical chains of spin-singlet dimers running around the tetragonal $c$ axis (VBC state), as observed in $\mathrm{MgTi}_{2} \mathrm{O}_{4}$. Each helix in the VBC state is ferroorbitally ordered (either $d_{x z}$ or $d_{y z}$ ) and adjacent helices have different orbital occupancy. We have also discussed the effect of an applied magnetic field and have shown that, at some critical value, it can destroy the dimer phase through a first- 


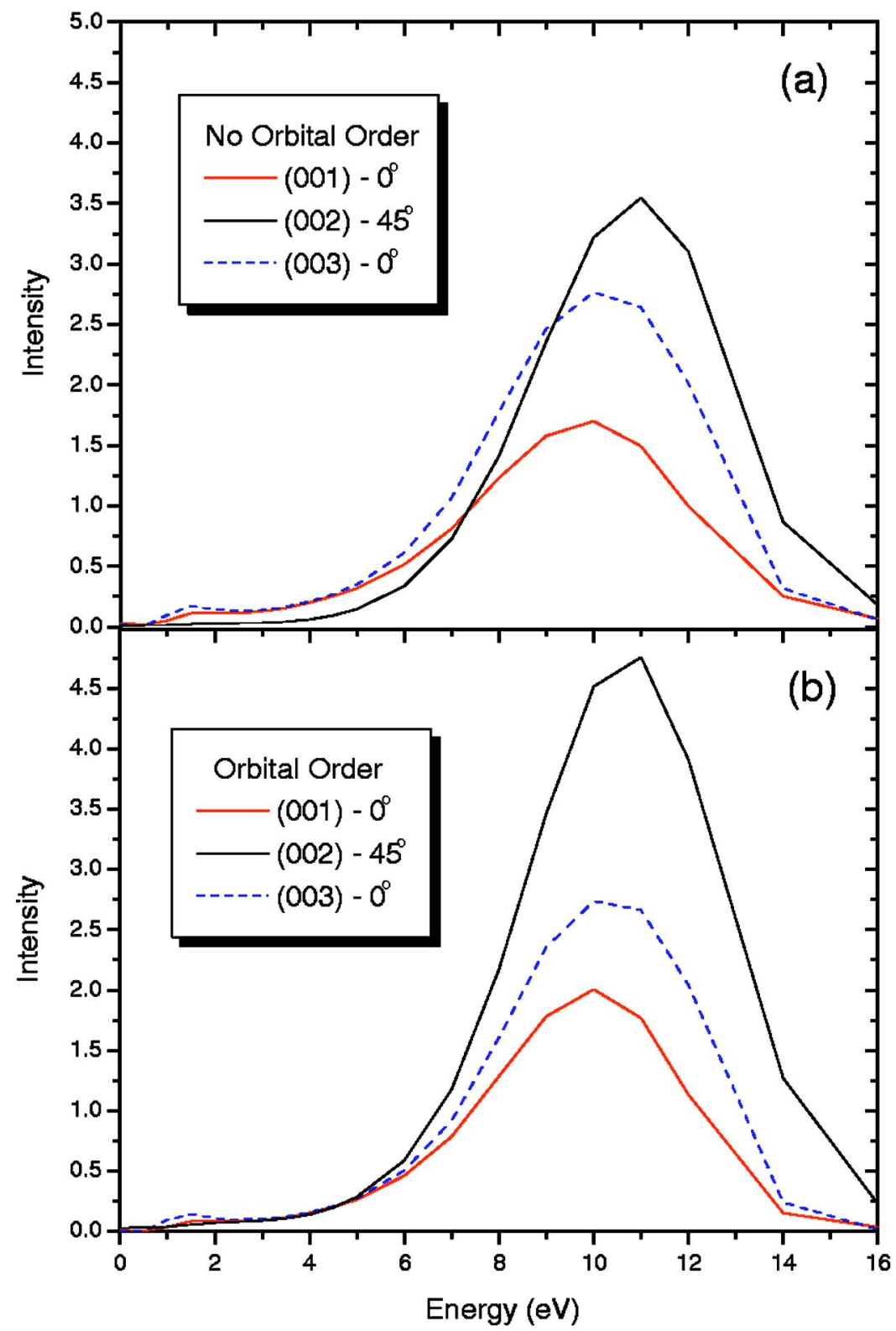

FIG. 7. (Color online) The RXS intensities for $(00 l)$ reflections $(l=1,2,3)$ without OO (a) and with OO (b). Angles refer to incoming $\sigma$ polarization: $\phi=0^{\circ}$ coincides with the cubic $x$ axis and $\phi=45^{\circ}$ with the tetragonal $x$ axis. The cluster radius is $3.3 \AA$. The reference energy is the Fermi level.

order transition to a FM phase, with a change of orbital order and the abrupt closure of the spin gap. Finally, we have proposed a RXS experiment to detect the predicted orbital ordering. We have performed a numerical simulation by means of the finite difference method ${ }^{18}$ and found out that the obtained $\mathrm{OO}$ can be experimentally revealed through the comparison of different RXS signals. In particular, an experimental setup that measures the (001), (002), and (003) Bragg-forbidden reflections keeping track of the relative intensity can be sufficient to determine whether the orbital order is present or not: If the three reflections have almost the same intensity, there is no OO, while if the (002) is about twice that of the others, this is the signature of the $\mathrm{OO}$ of the kind predicted by our theory.

\section{ACKNOWLEDGMENTS}

Several stimulating discussions with C.R. Natoli and C. Lacroix are gratefully acknowledged.

\section{APPENDIX A: THE “FULL” EFFECTIVE HAMILTONIAN}

In a previous Letter ${ }^{19}$ we have treated the effective Hamiltonian à la Kugel-Khomskii ${ }^{17}$ with $d d \sigma$ contributions only. In this appendix, for completeness, we take into account also the smaller terms proportional to the orbital overlaps of $d d \pi$ and $d d \delta$ type. In this case the hopping matrix is not diagonal. If we label planes and orbitals directions as $y z \equiv 1, x z \equiv 2$, and $x y \equiv 3$, the matrix elements can be expressed in the following form, given by Slater and Koster: ${ }^{35}$ 


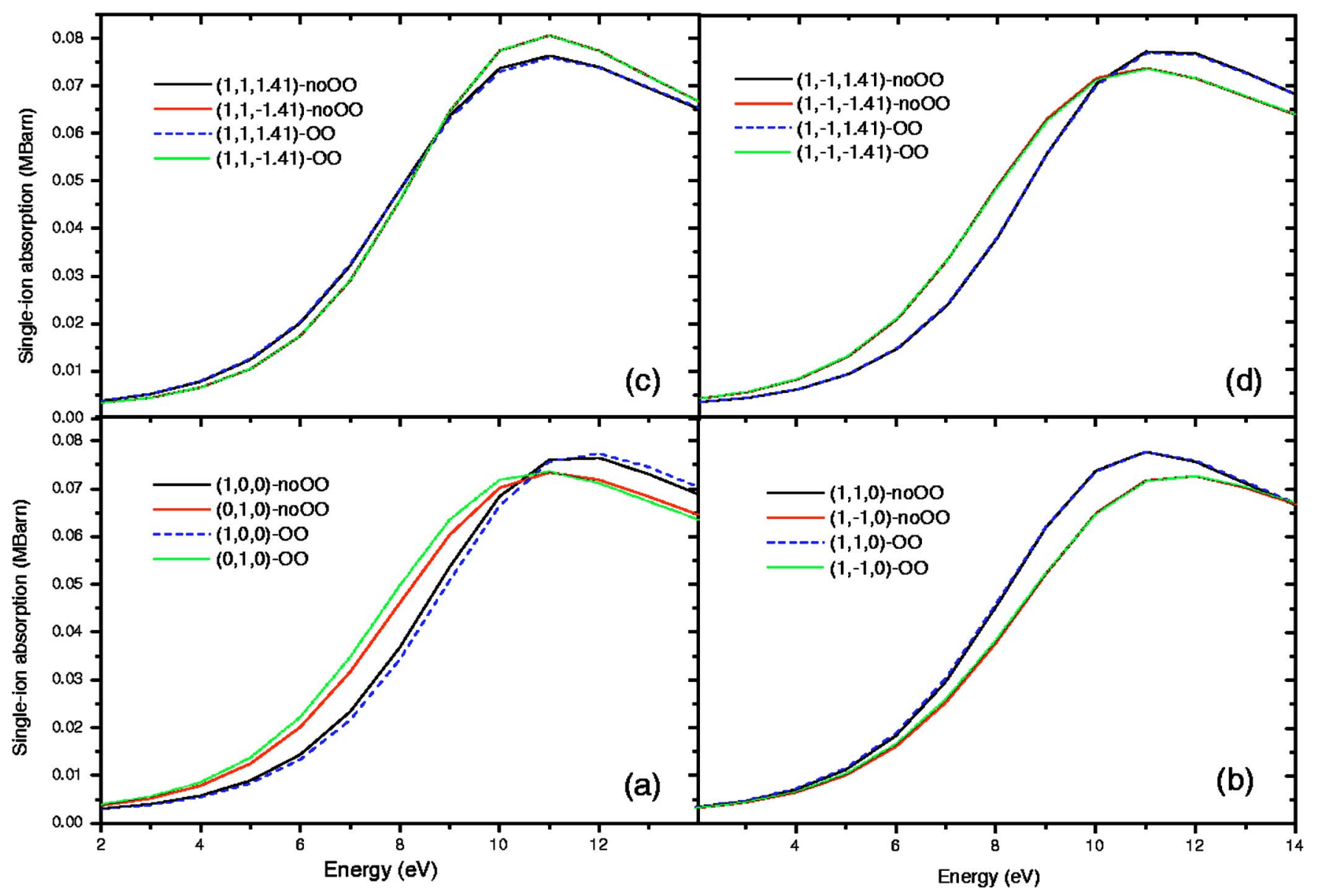

FIG. 8. (Color online) The absorption spectra for a $\mathrm{TiO}_{6}$ cluster in $\mathrm{MgTi}_{2} \mathrm{O}_{4}$. Directions of incident polarizations are expressed in the tetragonal frame. Energy zero is at the Fermi level. For each direction the comparison is made between the OO case and that without OO.

$$
\begin{gathered}
t_{i j(a)}^{a a}=3 / 4 d d \sigma+1 / 4 d d \delta, \\
t_{i j(a)}^{b b}=1 / 2 d d \pi+1 / 2 d d \delta, \\
t_{i j(a)}^{a b}=0, \\
t_{i j(a)}^{b c}=1 / 2 d d \pi-1 / 2 d d \delta,
\end{gathered}
$$

where $a, b$, and $c$ can be 1,2 , or 3 . Lower indices denote the plane $(a)$ where the bond $i j$ lies, and upper ones label the orbitals. For example, $t_{i j(3)}^{12}$ is the hopping matrix element from $y z$ to $x z$ orbitals along the bond $i j$ in the $x y$ plane.

Then, we introduce on-site transition orbital operators $T_{i}^{a b}$, which describe initial and final orbital states in the hopping process at site $i$. If there is no change in the orbital state at site $i$, then operators $T_{i}^{a a}$ are simply orbital projectors and can be written as $T_{i}^{11}=-\frac{1}{2} \tau_{i}^{z}\left(1-\tau_{i}^{z}\right), \quad T_{i}^{22}=\frac{1}{2} \tau_{i}^{z}\left(1+\tau_{i}^{z}\right)$, and $T_{i}^{33}$ $=\left(1+\tau_{i}^{z}\right)\left(1-\tau_{i}^{z}\right)$. Nondiagonal matrix elements of the transition orbital operator describe changes in the orbital state during the superexchange process: $T_{i}^{12}=\frac{1}{2} \tau_{i}^{+} \tau_{i}^{+}, T_{i}^{21}=\frac{1}{2} \tau_{i}^{-} \tau_{i}^{-}, T_{i}^{13}$ $=-(1 / \sqrt{2}) \tau_{i}^{+} \tau_{i}^{z}, T_{i}^{31}=-(1 / \sqrt{2}) \tau_{i}^{z} \tau_{i}^{-}, T_{i}^{23}=(1 / \sqrt{2}) \tau_{i}^{-} \tau_{i}^{z}$, and $T_{i}^{32}$ $=(1 / \sqrt{2}) \tau_{i}^{z} \tau_{i}^{+}$.
With these premises, the effective spin-orbital Hamiltonian can be written as

$$
\begin{aligned}
H_{\mathrm{eff}}= & -\frac{1}{U_{2}-J_{H}} \sum_{\langle i j\rangle}\left(\vec{S}_{i} \cdot \vec{S}_{j}+3 / 4\right) O_{i j}^{S} \\
& +\frac{1}{U_{2}+J_{H}} \sum_{\langle i j\rangle}\left(\vec{S}_{i} \cdot \vec{S}_{j}-1 / 4\right) O_{i j}^{S} \\
& +\sum_{\langle i j\rangle}\left(\vec{S}_{i} \cdot \vec{S}_{j}-1 / 4\right)\left(D_{1} O_{i j}^{D_{1}}+D_{2} O_{i j}^{D_{2}}\right),
\end{aligned}
$$

where the sum is restricted to the NN sites of the pyrochlore lattice. The first term in $H_{\text {eff }}$ describes ferromagnetic interactions, while second and third terms are antiferromagnetic. The explicit form of orbital contributions depends on the kind of intermediate excited states involved in the superexchange process: when the intermediate states have just singly-occupied orbitals $\left(O_{i j}^{S}\right)$, their expression is

$$
\begin{aligned}
O_{i j}^{S}= & \sum_{a=1}^{3} \sum_{b=1}^{3}\left(\sum_{\substack{c, d=1 \\
c \neq d}}^{3} t_{i j}^{a c} t_{j i}^{c b} T_{i}^{a b} T_{j}^{d d}+\sum_{\substack{c=1 \\
c \neq b}}^{3} t_{i j}^{a c} t_{j i}^{b a} T_{i}^{a a} T_{j}^{b c}\right) \\
& +\sum_{\substack{a, b=1 \\
a \neq b}}^{3} \sum_{\substack{c, d=1 \\
c \neq d}}^{3} t_{i j}^{a d} t_{j i}^{c b} T_{i}^{a b} T_{j}^{c d} .
\end{aligned}
$$


This orbital term is common to both FM and AFM bonds, whose difference resides in their energy denominators, $1 /\left(U_{2}-J_{H}\right)$ for the FM bond and $1 /\left(U_{2}+J_{H}\right)$ for the AFM.

The third term of the Hamiltonian describes the antiferromagnetic superexchange evolving through an intermediate excited state with double occupancy. The particular form with two energy denominators $\left(D_{1}\right.$ and $\left.D_{2}\right)$ and two corresponding orbital terms is originated by the fact that orbitally doubly occupied states are not eigenstates of the on-site multiband Hubbard Hamiltonian, ${ }^{36}$ due to interorbital double hopping. Exact eigenstates with single-orbital double occupancy are just linear combinations (with plus or minus sign) of two orbitally doubly occupied ionic states, and have two different energies, $U_{2}+J_{H}$ and $U_{2}+4 J_{H}$. If, in the superexchange process, the electron forms a double occupancy at some orbital on site $j$ and comes back from the same orbital, then the energy denominator is $D_{1}=2 / 3\left(U_{2}+J\right)+1 / 3\left(U_{2}\right.$ $+4 J)$. If, on the contrary, the orbital state on site $j$ changes during the hopping process, then the energy denominator is $D_{2}=-1 / 3\left(U_{2}+J\right)+1 / 3\left(U_{2}+4 J\right)$. The corresponding orbital contributions to $H_{\text {eff }}$ are

$$
\begin{gathered}
O_{i j}^{D_{1}}=\sum_{a, b, c=1}^{3} t_{i j}^{a b} t_{j i}^{b c} T_{i}^{a c} T_{j}^{b b}, \\
O_{i j}^{D_{2}}=\sum_{a, b=1}^{3} \sum_{\substack{c, d=1 \\
c \neq b}}^{3} t_{i j}^{a b} t_{j i}^{c d} T_{i}^{a d} T_{j}^{b c} .
\end{gathered}
$$

In the absence of $d d \pi$ and $d d \delta$ contributions, the hopping matrix connects only orbitals of the same type, $a$, which have nonzero overlap only in the corresponding $a$ plane. Then the effective spin-orbital Hamiltonian (A2) simplifies to the one we have obtained in Ref. 19 and which we deal with in Sec. II.

\section{APPENDIX B: MAGNETOELASTIC NORMAL MODES FOR A SINGLE TETRAHEDRON}

The normal modes of a single tetrahedron are a wellknown argument: yet, as we need their explicit expressions both here and in Sec. III, we prefer to review the basic results. Considering the small ionic displacements around the equilibrium positions, the coordinate of the four ions, when the origin is at the center of the tetrahedron, read $i_{1} \equiv\left(d_{0}\right.$ $\left.+x_{1}, d_{0}+y_{1}, d_{0}+z_{1}\right), i_{2} \equiv\left(-d_{0}+x_{2},-d_{0}+y_{2}, d_{0}+z_{2}\right), i_{3} \equiv\left(-d_{0}\right.$ $\left.+x_{3}, d_{0}+y_{3},-d_{0}+z_{3}\right)$, and $i_{4} \equiv\left(d_{0}+x_{4},-d_{0}+y_{4},-d_{0}+z_{4}\right)$. Assuming that the four ions are connected to each other with six equal springs of constant $k$, there will be an associated potential energy expressed as a bilinear form of the 12 displacements $x_{1}, \ldots, z_{4}$. The diagonalization of this form leads to the 12 normal modes of the tetrahedron, six of which are the zero-energy modes associated to the three global translations and the three global rotation, wihch we may neglect in the following.

The remaining six eigenvectors can be classified into a triplet $T$ mode, with eigenvalue $k_{t}=4 k$, a doublet $E$ mode, with eigenvalue $k_{e}=2 k$, and a singlet $S$ mode, with eigen- value $k_{s}=8 k$. They all correspond to a particular deformation of the tetrahedron: those of $T$ modes are shown in Fig. 5; those of the $E$ mode correspond to a local tetragonal or orthorhombic distortion (see, e.g., Ref. 4); and that of the $S$ mode is the "breathing" mode, i.e., a volume expansion or contraction that does not change the tetrahedron shape. If we introduce the stretches of each bond, $\delta d_{i j}$, in terms of the ionic displacement coordinates, it is then possible to express normal coordinates in terms of the latter.

$$
\begin{aligned}
& \delta d_{12}=\frac{1}{\sqrt{2}}\left(x_{1}-x_{2}+y_{1}-y_{2}\right), \\
& \delta d_{34}=\frac{1}{\sqrt{2}}\left(x_{4}-x_{3}+y_{3}-y_{4}\right), \\
& \delta d_{13}=\frac{1}{\sqrt{2}}\left(x_{1}-x_{3}+z_{1}-z_{3}\right), \\
& \delta d_{24}=\frac{1}{\sqrt{2}}\left(x_{4}-x_{2}+z_{2}-z_{4}\right), \\
& \delta d_{14}=\frac{1}{\sqrt{2}}\left(y_{1}-y_{4}+z_{1}-z_{4}\right), \\
& \delta d_{23}=\frac{1}{\sqrt{2}}\left(y_{3}-y_{2}+z_{2}-z_{3}\right),
\end{aligned}
$$

and

$$
\begin{gathered}
\theta_{s}=\frac{1}{2 \sqrt{6}}\left(\delta d_{12}+\delta d_{34}+\delta d_{13}+\delta d_{24}+\delta d_{14}+\delta d_{23}\right), \\
\theta_{e 1}=\frac{1}{2}\left(\delta d_{13}+\delta d_{24}-\delta d_{14}-\delta d_{23}\right), \\
\theta_{e 2}=\frac{1}{2 \sqrt{3}}\left(2 \delta d_{12}+2 \delta d_{34}-\delta d_{13}-\delta d_{24}-\delta d_{14}-\delta d_{23}\right), \\
\theta_{t 1}=\frac{1}{2}\left(\delta d_{12}-\delta d_{34}\right), \\
\theta_{t 2}=\frac{1}{2}\left(\delta d_{13}-\delta d_{24}\right), \\
\theta_{t 3}=\frac{1}{2}\left(\delta d_{14}-\delta d_{23}\right),
\end{gathered}
$$

where $\theta_{s}, \theta_{e i}$, and $\theta_{t i}$ are, respectively, singlet, doublet, and triplet normal modes of the tetrahedron group.

The inverted relations are

$$
\begin{gathered}
\delta d_{12}=\frac{2}{\sqrt{6}} \theta_{s}+\frac{1}{\sqrt{3}} \theta_{e 2}+\theta_{t 1}, \\
\delta d_{34}=\frac{2}{\sqrt{6}} \theta_{s}+\frac{1}{\sqrt{3}} \theta_{e 2}-\theta_{t 1}, \\
\delta d_{13}=\frac{2}{\sqrt{6}} \theta_{s}-\frac{1}{2 \sqrt{3}} \theta_{e 2}+\frac{1}{2} \theta_{e 1}+\theta_{t 2},
\end{gathered}
$$




$$
\begin{aligned}
& \delta d_{24}=\frac{2}{\sqrt{6}} \theta_{s}-\frac{1}{2 \sqrt{3}} \theta_{e 2}+\frac{1}{2} \theta_{e 1}-\theta_{t 2}, \\
& \delta d_{14}=\frac{2}{\sqrt{6}} \theta_{s}-\frac{1}{2 \sqrt{3}} \theta_{e 2}-\frac{1}{2} \theta_{e 1}+\theta_{t 3}, \\
& \delta d_{23}=\frac{2}{\sqrt{6}} \theta_{s}-\frac{1}{2 \sqrt{3}} \theta_{e 2}-\frac{1}{2} \theta_{e 1}-\theta_{t 3} .
\end{aligned}
$$

The spin-lattice interaction Hamiltonian becomes, then,

$$
\begin{aligned}
H_{s l}= & -\frac{2}{\sqrt{6}} g \theta_{s}\left(E_{12}+E_{34}+E_{13}+E_{24}+E_{14}+E_{23}\right)-\frac{1}{2} g \theta_{e 1}\left(E_{13}\right. \\
& \left.+E_{24}-E_{14}-E_{23}\right)-\frac{1}{2 \sqrt{3}} g \theta_{e 2}\left(2 E_{12}+2 E_{34}-E_{13}-E_{24}\right. \\
& \left.-E_{14}-E_{23}\right)-g \theta_{t 1}\left(E_{12}-E_{34}\right)-g \theta_{t 2}\left(E_{13}-E_{24}\right) \\
& -g \theta_{t 3}\left(E_{14}-E_{23}\right),
\end{aligned}
$$

where $E_{i j}$ denotes the energy of the $i j$ bond in $J$ units and $\left.g \equiv[\partial J(d) / \partial d]\right|_{d=d_{0}}$, with $d_{0}$ the equilibrium distance.

In this way it is straightforward to have the energy gain related to each mode of our spin-orbital model. Treating separately all the cases of topologically distinct tetrahedra, we obtain the following:

(1) A tetrahedron with two spin singlets located at bonds $d_{12}$ and $d_{34}$. Superexchange energies are $E_{12}=E_{34}=-4$ and $E_{13}=E_{24}=E_{14}=E_{23}=0$. The global magnetoelastic Hamiltonian $H_{m e A}=H_{s l A}+H_{e l A}$ is

$$
H_{m e A}=\frac{16}{\sqrt{6}} g \theta_{s}+\frac{1}{2} k_{s} \theta_{s}^{2}+\frac{8}{\sqrt{3}} g \theta_{e 2}+\frac{1}{2} k_{e} \theta_{e 2}^{2} .
$$

As each normal mode is independent of the other, it is possible to minimize with respect to the two variables separately and get the energy minima: $E_{s m}=-64 g^{2} / 3 k_{s}$, and $E_{e 2 m}=-32 g^{2} / 3 k_{e}$. The normal modes corresponding to these minima are $\theta_{s m}=-16 g / \sqrt{6} k_{s}$ and $\theta_{e 2 m}=-8 g / \sqrt{3} k_{e}$. As $k_{s}=2 k_{t}=4 k_{e}=8 \mathrm{k}$, the global minimum is $E_{m}=-8 \mathrm{~g}^{2} / \mathrm{k}$.

(2) $B_{1}$ tetrahedron with superexchange energies $E_{14}$ $=E_{24}=-1, E_{13}=-4$, and $E_{23}=E_{12}=E_{34}=0$. The global magnetoelastic Hamiltonian is

$$
\begin{aligned}
H_{m e B 2}= & \frac{12}{\sqrt{6}} g \theta_{s}+\frac{1}{2} k_{s} \theta_{s}^{2}+2 g \theta_{e 1}+\frac{1}{2} k_{e} \theta_{e 1}^{2}-\sqrt{3} g \theta_{e 2}+\frac{1}{2} k_{e} \theta_{e 2}^{2} \\
& +3 g \theta_{t 2}+\frac{1}{2} k_{t} \theta_{t 2}^{2}+g \theta_{t 3}+\frac{1}{2} k_{t} \theta_{t 3}^{2} .
\end{aligned}
$$

The energy minima are $E_{s m}=-12 g^{2} / k_{s}, E_{e 1 m}=-2 g^{2} / k_{e}$, $E_{e 2 m}=-3 g^{2} / 2 k_{e}, E_{t 2 m}=-9 g^{2} / 2 k_{t}$, and $E_{t 3 m}=-g^{2} / 2 k_{t}$. The normal modes corresponding to these minima are $\theta_{s m}$ $=-12 g / \sqrt{6} k_{s}, \theta_{e 1 m}=-2 g / k_{e}, \theta_{e 2 m}=\sqrt{3} g / k_{e}, \theta_{t 2 m}=-3 g / k_{t}$, and $\theta_{t 3 m}=-g / k_{t}$. The global minimum is $E_{m}=-4.5 g^{2} / k$.

(3) $B_{2}$ tetrahedron with superexchange energies $E_{14}$ $=E_{12}=-1, E_{13}=-4$, and $E_{23}=E_{24}=E_{34}=0$. The global magnetoelastic Hamiltonian is

$$
\begin{aligned}
H_{m e B 3}= & \frac{12}{\sqrt{6}} g \theta_{s}+\frac{1}{2} k_{s} \theta_{s}^{2}+\frac{3}{2} g \theta_{e 1}+\frac{1}{2} k_{e} \theta_{e 1}^{2}-\frac{\sqrt{3}}{2} g \theta_{e 2}+\frac{1}{2} k_{e} \theta_{e 2}^{2} \\
& +g \theta_{t 1}+\frac{1}{2} k_{t} \theta_{t 1}^{2}+4 g \theta_{t 2}+\frac{1}{2} k_{t} \theta_{t 2}^{2}+g \theta_{t 3}+\frac{1}{2} k_{t} \theta_{t 3}^{2} .
\end{aligned}
$$

The energy minima are $E_{s m}=-12 g^{2} / k_{s}, E_{e 1 m}=-9 g^{2} / 8 k_{e}$, $E_{e 2 m}=-3 g^{2} / 8 k_{e}, \quad E_{t 2 m}=-8 g^{2} / k_{t}$, and $E_{t 1 m}=E_{t 3 m}=-g^{2} / 2 k_{t}$. The normal modes corresponding to these minima are $\theta_{s m}=-12 g / \sqrt{6} k_{s}, \quad \theta_{e 1 m}=-3 g / 2 k_{e}, \quad \theta_{e 2 m}=\sqrt{3} g / 2 k_{e}, \quad \theta_{t 2 m}$ $=-4 g / k_{t}$, and $\theta_{t 1 m}=\theta_{t 3 m}=-g / k_{t}$. The global minimum is again $E_{m}=-4.5 g^{2} / \mathrm{k}$.

(4) $B_{3}$ tetrahedron with superexchange energies $E_{14}$ $=E_{23}=-1, E_{13}=-4$, and $E_{24}=E_{12}=E_{34}=0$. The global magnetoelastic Hamiltonian is

$$
\begin{aligned}
H_{m e B 1}= & \frac{12}{\sqrt{6}} g \theta_{s}+\frac{1}{2} k_{s} \theta_{s}^{2}+g \theta_{e 1}+\frac{1}{2} k_{e} \theta_{e 1}^{2} \\
& -\sqrt{3} g \theta_{e 2}+\frac{1}{2} k_{e} \theta_{e 2}^{2}+4 g \theta_{t 2}+\frac{1}{2} k_{t} \theta_{t 2}^{2} .
\end{aligned}
$$

The energy minima are $E_{s m}=-12 g^{2} / k_{s}, E_{e 1 m}=-g^{2} / 2 k_{e}$, $E_{e 2 m}=-3 g^{2} / 2 k_{e}$, and $E_{t 2 m}=-8 g^{2} / k_{t}$. The normal modes corresponding to these minima are: $\theta_{s m}=-12 g / \sqrt{6} k_{s}, \theta_{e 1 m}$ $=-g / k_{e}, \theta_{e 2 m}=\sqrt{3} g / k_{e}$ and $\theta_{t 2 m}=-4 g / k_{t}$. The global minimum is still $E_{m}=-4.5 g^{2} / \mathrm{k}$.

Due to the energy gain in the triplet $t_{2}$ mode, there is a shortening of the bond where the singlet is located, as it can be seen by substituting back the previous expressions for normal minima in Eqs. (B3) and (B4).

It is worthwhile to note that even though all three $B$ tetrahedra have the same magnetoelastic energy, the corresponding bond distortions are different, and thus the three configurations match one another differently when forced in the global fcc cell.

(5) $C_{1}$ tetrahedron [the one shown in Fig. 2(e)] with superexchange energies $E_{12}=E_{34}=0$ and $E_{13}=E_{24}=E_{14}=E_{23}$ $=-1$. The global magnetoelastic Hamiltonian is

$$
H_{m e C 1}=\frac{8}{\sqrt{6}} g \theta_{s}+\frac{1}{2} k_{s} \theta_{s}^{2}-\frac{2}{\sqrt{3}} g \theta_{e 2}+\frac{1}{2} k_{e} \theta_{e 2}^{2} \text {. }
$$

The energy minima are $E_{s m}=-16 g^{2} / 3 k_{s}$, and $E_{e 2 m}$ $=-2 g^{2} / 3 k_{e}$. The normal modes corresponding to these minima are $\theta_{s m}=-8 g / \sqrt{6} k_{s}$, and $\theta_{e 2 m}=2 g / \sqrt{3} k_{e}$. The global minimum is $E_{m}=-g^{2} / \mathrm{k}$.

(6) $C_{2}$ tetrahedron (not shown in Fig. 2). They are characterized by four $b_{1}$, one $b_{2}$, and one $b_{3}$ bonds, with superexchange energies $E_{23}=E_{24}=0$ and $E_{13}=E_{14}=E_{12}=E_{34}=-1$. The global magnetoelastic Hamiltonian is

$$
\begin{aligned}
H_{m e C 2}= & \frac{8}{\sqrt{6}} g \theta_{s}+\frac{1}{2} k_{s} \theta_{s}^{2}+\frac{1}{\sqrt{3}} g \theta_{e 2}+\frac{1}{2} k_{e} \theta_{e 2}^{2}+g \theta_{t 2}+\frac{1}{2} k_{t} \theta_{t 2}^{2} \\
& +g \theta_{t 3}+\frac{1}{2} k_{t} \theta_{t 3}^{2} .
\end{aligned}
$$

The energy minima are $E_{s m}=-16 g^{2} / 3 k_{s}, E_{e 2 m}=-g^{2} / 6 k_{e}$, and $E_{t 2 m}=E_{t 3 m}=-g^{2} / 2 k_{t}$. The normal modes corresponding to 
these minima are $\theta_{s m}=-8 g / \sqrt{6} k_{s}, \theta_{e 2 m}=-g / \sqrt{3} k_{e}$, and $\theta_{t 2 m}$ $=\theta_{t 3 m}=-g / k_{t}$. As in the case of $B_{i}$ tetrahedra, the partial contributions of each mode compensate and the global minimum is, as for $C_{1}$ case, $E_{m}=-g^{2} / k$.
*Also at E. Andronikashvili Institute of Physics, Georgian Academy of Sciences, Tbilisi, Gorgia.

${ }^{1}$ A. P. Ramirez, in Handbook of Magnetic Materials, edited by K. H. J. Buschow (North-Holland, Amsterdam, 2001).

2 J. Villain, R. Bidaux, J. P. Carton, and R. Conte, J. Phys. (Paris) 41, 1263 (1980).

${ }^{3}$ Y. Yamashita and K. Ueda, Phys. Rev. Lett. 85, 4960 (2000).

${ }^{4}$ O. Tchernyshyov, R. Moessner, and S. L. Sondhi, Phys. Rev. B 66, 064403 (2002).

${ }^{5}$ R. Moessner and J. T. Chalker, Phys. Rev. Lett. 80, 2929 (1998); Phys. Rev. B 58, 12049 (1998).

${ }^{6}$ B. Canals and C. Lacroix, Phys. Rev. Lett. 80, 2933 (1998); H. Tsunetsugu, Phys. Rev. B 65, 024415 (2001).

${ }^{7}$ H. F. Pen, J. Vanden Brink, D. I. Khomskii, and G. A. Sawatzley, Phys. Rev. Lett. 78, 1323 (1997).

${ }^{8}$ H. Tsunetsugu and Y. Motome, Phys. Rev. B 68, 060405(R) (2003); O. Tchernyshyov, Phys. Rev. Lett. 93, 157206 (2004).

${ }^{9}$ M. Isobe and Y. Ueda, J. Phys. Soc. Jpn. 71, 1848 (2002).

${ }^{10}$ M. Schmidt, W. Ratcliff II, P. G. Radoelli, K. Refson, N. M. Harrison, and S. W. Cheong, Phys. Rev. Lett. 92, 056402 (2004).

${ }^{11}$ We neglect here the effects of the smaller trigonal field in the $t_{2 g}$ manifold.

${ }^{12}$ L. F. Feiner, A. M. Oles, and J. Zaanen, Phys. Rev. Lett. 78, 2799 (1997).

${ }^{13}$ I. S. Elfimov, V. I. Anisimov, and G. A. Sawatzky, Phys. Rev. Lett. 82, 4264 (1999).

${ }^{14}$ M. Benfatto, Y. Joly, and C. R. Natoli, Phys. Rev. Lett. 83, 636 (1999).

${ }^{15}$ M. Takahashi, J. Igarashi and P. Fulde, J. Phys. Soc. Jpn. 68, 6530 (1999).

${ }^{16}$ S. Di Matteo, T. Chatterji, Y. Joly, A. Stunault, J. A. Paixao, R. Suryanarayanan, G. Dhalenne, and A. Revcolevschi, Phys. Rev. B 68, 024414 (2003).

${ }^{17}$ K. I. Kugel and D. I. Khomskii, Usp. Fiz. Nauk 136, 621 (1982), [Sov. Phys. Usp. 25, 231 (1982)].

${ }^{18}$ Y. Joly, Phys. Rev. B 63, 125120 (2001); the computer program can be freely downloaded at the web address: http://wwwcristallo.grenoble.cnrs.fr/simulation

${ }^{19}$ S. Di Matteo, G. Jackeli, C. Lacroix, and N. B. Perkins, Phys. Rev. Lett. 93, 077208 (2004).

${ }^{20}$ J. Matsuno, A. Fujimori, and L. F. Mattheiss, Phys. Rev. B 60, 1607 (1999).

${ }^{21}$ T. Mizokawa and A. Fujimori, Phys. Rev. B 54, 5368 (1996).

${ }^{22}$ M. Takhashi, Thermodynamics of One-Dimensional Solvable Models (Cambridge University Press, Cambridge, 1999).

${ }^{23}$ B. Canals and C. Lacroix, Phys. Rev. B 61, 1149 (2000).

${ }^{24}$ If a complex linear combination is considered, there could be an associated spin-orbit energy gain. However, we have checked that the energy loss related to the breakdown of the spin-singlet state exceeds by far the possible spin-orbit energy gain.

${ }^{25}$ In the case of ferro-orbital order there are in principle two $b_{0}$ bonds every tetrahedron. Yet, when the whole pyrochlore lattice is considered, only one of the two can be "active" as a spinsinglet.

${ }^{26}$ W. Harrison, Electronic Structure and the Properties of Solids, (Dover, New York, 1989).

${ }^{27}$ Y. Murakami, H. Kawada, H. Kawata, M. Tanaka, T. Arima, Y. Moritomo, and Y. Tokura, Phys. Rev. Lett. 80, 1932 (1998); 81, 582 (1998).

${ }^{28}$ S. Ishihara and S. Maekawa, Phys. Rev. Lett. 80, 3799 (1998).

${ }^{29}$ Due to the damping term $\Gamma$, the signal at a given energy involves an integral around that energy of width $\Gamma$ [see Eq. (10)].

${ }^{30}$ C. W. M. Castleton and M. Altarelli, Phys. Rev. B 62, 1033 (2000).

${ }^{31}$ This value is estimated from Ref. 14.

${ }^{32}$ M. Blume, in Resonant Anomalous X-ray Scattering, edited by G. Materlik, J. Sparks, and K. Fischer (Elsevier, Amsterdam, 1994), p. 495.

${ }^{33}$ International Tables for Cristallography, 5th ed., edited by Theo Hahn (Kluwer, Dordrecht, 2002).

${ }^{34}$ S. Di Matteo and C. R. Natoli, J. Synchrotron Radiat. 9, 9 (2002).

${ }^{35}$ J. C. Slater and G. F. Koster, Phys. Rev. 94, 1498 (1954).

${ }^{36}$ S. Di Matteo, N. B. Perkins, and C. R. Natoli, Phys. Rev. B 65, 054413 (2002) 